\title{
ANALYSIS OF A DISLOCATION MODEL FOR EARTHQUAKES
}

\author{
BY \\ JING LIU (Department of Mathematical Sciences, Carnegie Mellon University, Pittsburgh, \\ Pennsylvania 15213), \\ XIN YANG LU (Department of Mathematics and Statistics, McGill University, Montreal, Canada), \\ AND \\ NOEL J. WALKINGTON (Department of Mathematical Sciences, Carnegie Mellon University, \\ Pittsburgh, Pennsylvania 15213)

\begin{abstract}
Approximation of problems in linear elasticity having small shear modulus in a thin region is considered. Problems of this type arise when modeling ground motion due to earthquakes where rupture occurs in a thin fault. It is shown that, under appropriate scaling, solutions of these problems can be approximated by solutions of a limit problem where the fault region is represented by a surface. In a numerical context this eliminates the need to resolve the large deformations in the fault; a numerical example is presented to illustrate efficacy of this strategy.
\end{abstract}

1. Introduction. Models used to simulate ground motion during an earthquake frequently represent the sub-surface as a union of linearly elastic materials separated by thin (fault) regions within which large deformations (rupture) occur. Below we analyze limiting models which circumvent the numerical difficulties encountered with direct simulation of these models which arise when very fine meshes are required to resolve the large deformations in the fault region. The fault region in reduced models is represented as a surface and the rupture is realized as discontinuities in certain components of the solution. Figures 1 and 2 illustrate these issues; the fine mesh in Figure 1 is unnecessary when the large shear across the fault is represented as the discontinuity in the horizontal displacement shown in Figure 2. Theorems 2.1 and 3.1 justify this approach for a certain class of these models by establishing that their solutions converge to the solution of a reduced problem as the width of the fault tends to zero.

Received April 21, 2016.

2010 Mathematics Subject Classification. Primary 86A17, 74S05, 49J45.

The third author was supported in part by National Science Foundation grants DMS-1418991 and DMREF-1434734. This work was also supported by the NSF through the Center for Nonlinear Analysis. E-mail address: jingliu1@andrew.cmu.edu

E-mail address: xinyang.lu@mcgill.ca

E-mail address: noelw@andrew.cmu.edu 

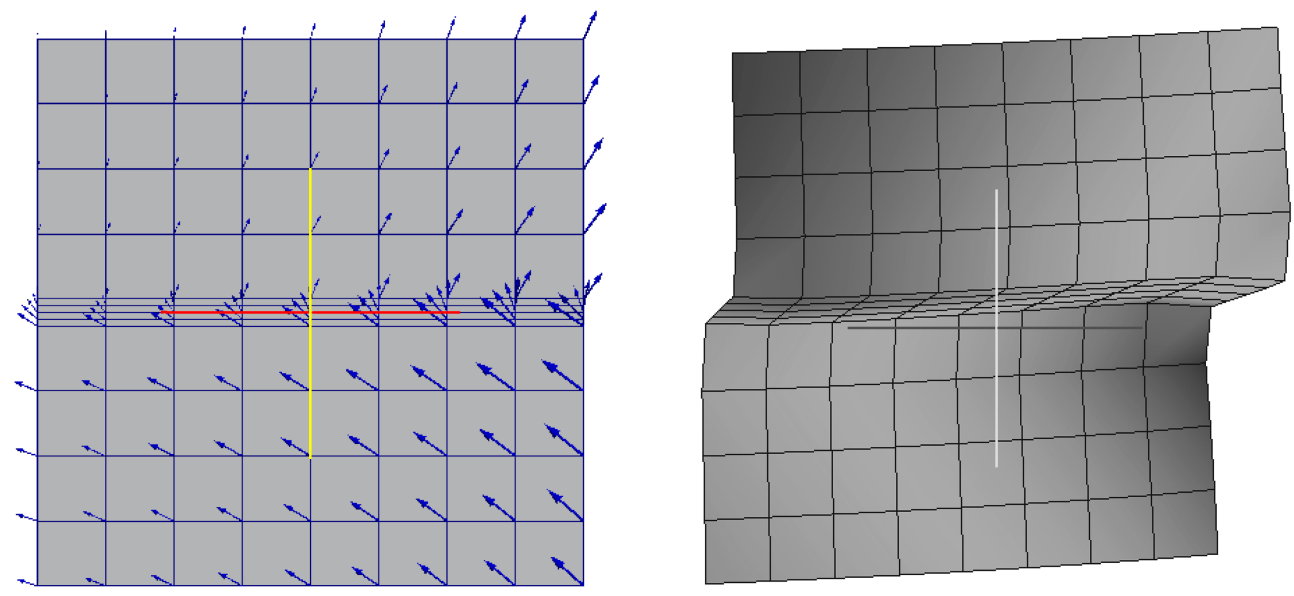

FIG. 1. Solution with deformation resolved.
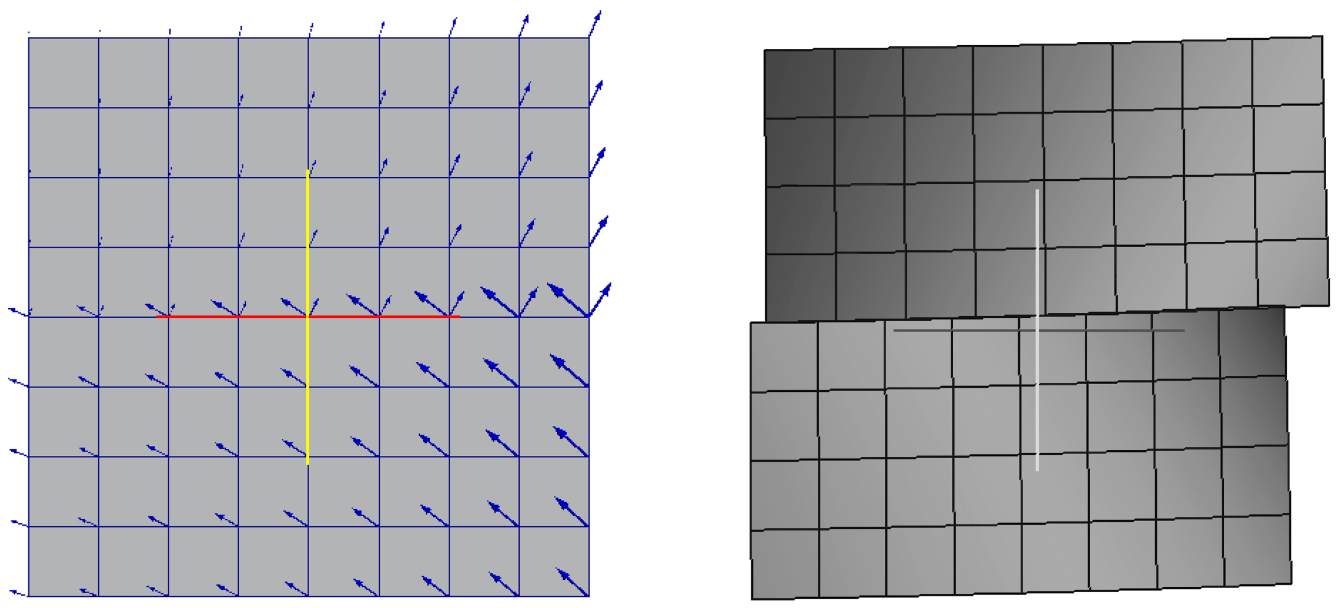

FIG. 2. Solution of the limit problem.

In the Appendix we show that a solution of the three dimensional rupture model proposed in 29] can be found by considering a cross section $\Omega=(-1,1) \times(-1,1)$ of a sub-surface region containing a horizontal fault $S_{\epsilon}=(-1,1) \times(-\epsilon / 2, \epsilon / 2)$ of width $\epsilon>0$. The balance of momentum takes the classical form

$$
\rho u_{t t}-\operatorname{div}(T)=\rho f \quad \text { on } \quad(0, T) \times \Omega,
$$

where $u(t, x) \in \mathbb{R}^{2}$ and $\rho(t, x)>0$ represent the displacement and density of the medium, $f(t, x) \in \mathbb{R}^{2}$ the force per unit mass, and $T$ is the (Cauchy) stress tensor. The sub-surface strata is taken to be isotropic so that away from the fault the stress takes the form

$$
T=2 \mu D(u)+\lambda \operatorname{div}(u) I, \quad \text { in } \Omega \backslash \bar{S}_{\epsilon},
$$


where $D(u)=(1 / 2)\left(\nabla u+(\nabla u)^{\top}\right)$ and $\mu=\mu(x)$ and $\lambda=\lambda(x)$ are the shear and bulk moduli. In the fault the stress is given by

$$
T=\left[\begin{array}{cc}
(2 \mu+\lambda) u_{1 x}+\lambda u_{2 y} & \mu\left(\epsilon\left(u_{2 x}+u_{1 y}\right)-\gamma\right) \\
\mu\left(\epsilon\left(u_{2 x}+u_{1 y}\right)-\gamma\right) & (2 \mu+\lambda) u_{2 y}+\lambda u_{1 x}
\end{array}\right] \quad \text { in } S_{\epsilon},
$$

where $\gamma=\gamma(t, x)$ models the permanent deformation due to damage, defects, and healing in the fault [29], and evolves according to

$$
(1 / \beta) \gamma_{t}+\hat{\eta} \gamma-T_{12}-\nu \gamma_{x x}=0, \quad \text { in } S_{\epsilon} .
$$

This model of rupture was inspired by the plasticity theories developed in [1, 3] where the coefficients $\beta, \hat{\eta}$ and $\nu$ are typically nonlinear functions of $\gamma$ and its derivatives. To date there is no satisfactory mathematical theory for these models of nonlinear elasticity [2], so for the analysis below we assume that the coefficients in (44) are specified which, for example, would be the case for one step of a linearly implicit time stepping scheme for the fully nonlinear problem. In this context we address the following problems.

Strain Energy: If $\gamma(x)$ is specified (or more generally $\gamma_{\epsilon} \rightarrow \gamma$ sufficiently strongly) we verify that the strain energies for the stationary problem (1)-(3) converge to the limiting energy

$$
I(u)=\frac{1}{2} \int_{\Omega \backslash S_{0}} 2 \mu|D(u)|^{2}+\lambda \operatorname{div}(u)^{2}+\frac{1}{2} \int_{S_{0}} \mu\left(\left[u_{1}\right]-\gamma\right)^{2},
$$

where $\left[u_{1}(x)\right]$ denotes the jump in the first (horizontal) component of $u$ across the line $S_{0}=(-1,1) \times\{0\}$. The corresponding Euler Lagrange operator is then

$$
-\operatorname{div}(T) \quad \text { on } \Omega \backslash S_{0} \quad \text { with } \quad[T n]=0 \text { and } T_{12}=\mu\left(\left[u_{1}\right]-\gamma\right) \quad \text { on } S_{0},
$$

where $T=2 \mu D(u)+\lambda \operatorname{div}(u) I$ and $[T n]$ is the jump of the traction across $S_{0}$ with $n=(0,1)^{\top}$ and $T_{12}$ its first component.

This shows that the scaling introduced in (3) is the "mathematically interesting" case for which a nontrivial limit exists. With different scalings the equations for $u$ and $\gamma$ either decouple (the last term in the energy vanishes) or lock, $\left[u_{1}\right]=\gamma$, in the limit. The limiting energy for the coupled stationary problem (11)-(41) is

$$
I(u, \gamma)=\frac{1}{2} \int_{\Omega \backslash S_{0}} 2 \mu|D(u)|^{2}+\lambda \operatorname{div}(u)^{2}+\frac{1}{2} \int_{S_{0}} \mu\left(\left[u_{1}\right]-\gamma\right)^{2}+\left(\hat{\eta} \gamma^{2}+\nu \gamma_{x}^{2}\right) .
$$

We omit the proof since the proof is a routine extension of the ideas used for the uncoupled problem. Examples of numerical solutions to both the uncoupled and coupled problems are presented in Section 4 .

Evolutionary Problem: In Section 3 we show that solutions of the coupled system (11) -(4) converge to a limit which satisfies the reduced system,

$$
\rho u_{t t}-\operatorname{div}(T)=\rho f \quad \text { on } \Omega \backslash S_{0},
$$

with $T=2 \mu D(u)+\lambda \operatorname{div}(u) I$, and

$$
[T n]=0, \quad T_{12}=\mu\left(\left[u_{1}\right]-\gamma\right), \text { and }(1 / \beta) \gamma_{t}+\hat{\eta} \gamma-T_{12}-\nu \gamma_{x x}=0 \quad \text { on } S_{0} .
$$


For definiteness we consider displacement boundary conditions $u(., \pm 1)=0$ on the top and bottom of $\Omega$ and traction free boundary data on the sides; $T( \pm 1,) n=$.0 , and $\nu \gamma_{x}( \pm 1,)=$.0 . We omit analogous results for other boundary conditions which are routine technical extensions of the proof techniques presented below. The same energy and limiting problem are obtained with the "engineering approximation" utilized in 29] where the shear stress $T_{12}(x, y)$ in the equation (4) is approximated by its average $\bar{T}_{12}(x)$ across $S_{\epsilon}$ so that $\gamma$ depends only upon $x$,

$$
(1 / \beta) \gamma_{t}+\hat{\eta} \gamma-\bar{T}_{12}-\nu \gamma_{x x}=0, \quad \text { where } \quad \bar{T}_{12}(t, x)=\frac{1}{\epsilon} \int_{-\epsilon / 2}^{\epsilon / 2} T_{12}(t, x, y) d y .
$$

The ideas presented below extend directly to the analysis of this variation of the problem.

1.1. Overview of related results. The modeling and prediction of material failure is a notoriously difficult problem with a long history. Broadly viewed, the onset of failure is modeled either by crack formation (brittle failure) or modifications of elasticity theory to admit plastic deformation (ductile failure). Equations (11)-(4) combine these approaches in the sense that a "crack like" fault region is known a priori; however, the material response in this zone is inspired by models of dislocation motion in ductile materials. This contrasts with "cohesive zone" models where friction laws complement the usual jump condition, $[T n]=0$, and kinematic compatibility condition, $\left[u_{2}\right] \geq 0$, (or $\left[u_{2}\right]=0$ on a fault deep underground). A detailed development of this model and comparison with, and references to, alternative models may be found in [29, Section 8.4].

A major challenge when modeling of brittle failure is predicting nucleation and propagation paths of the cracks [22]. To date the most mathematically complete approach to this issue is the variational technique initiated by Ambrosio and Braides [5], which uses spaces of bounded variation, and the corresponding extension to evolution problems by Francfort and Marigo [17] using the concept of minimizing movements [15]. This has resulted in a large body of mathematical work [4, 6, 10, 14, 19] and numerical schemes [7, 9, 16, 23]. To date models based upon these variational techniques do not include friction at the crack sites or restrict the relative motion to prevent interpenetration.

Almost all models of ductile failure are based upon the premise that the permanent macroscopic deformations of ductile materials result from the motion of dislocations through a microscopic crystalline lattice [21]. However, passage from the microscopic description to a macroscopic model with predictive capability requires a substantial amount of phenomenological input [1,20]. Again the most mathematically complete results for these models involve variational techniques and quasi-static formulations to construct minimizing movements $[13,24,28$. The analogue of the friction laws in this context is an assumption on the rate independence of the dissipation which gives rise to energies with linear growth for which minimizers have gradients of bounded deformation [12, 18, 25].

1.2. Notation $\&$ function spaces. Standard notation is adopted for the Lebesgue spaces, $L^{p}(\Omega)$, and the Sobolev space $H^{1}(\Omega)$. Solutions of evolution equations will be viewed as functions from $[0, T]$ into these spaces, and we adopt the usual notion, $L^{2}\left[0, T ; H^{1}(\Omega)\right], C\left[0, T ; H^{1}(\Omega)\right]$, etc., to indicate the temporal regularity. Strong and weak convergence in these spaces is denoted as $u^{\epsilon} \rightarrow u$ and $u^{\epsilon} \rightarrow u$ respectively. 
Divergences of vector and matrix valued functions are $\operatorname{denoted} \operatorname{div}(u)=u_{i, i}$ and $\operatorname{div}(T)_{i}=T_{i j, j}$ respectively. Here indices after the comma represent partial derivatives and the summation convention is used. Gradients of vector valued quantities are interpreted as matrices, $(\nabla u)_{i j}=u_{i, j}$, and the symmetric part of the gradient is written as $D(u)$. Inner products are typically denoted as pairings $(.,$.$) or, for clarity, the dot$ product of two vectors $v, w \in \mathbb{R}^{d}$ may be written as $v . w=v_{i} w_{i}$ and the Frobenious inner product of two matrices $A, B \in \mathbb{R}^{d \times d}$ as $A: B=A_{i j} B_{i j}$.

The following notation is used to characterize the dependence upon $\epsilon$ of the elastic and fault regions.

NotATion 1.1. Let $\Omega=(-1,1)^{2}$ and $0<\epsilon<1 / 2$.

(1) The fault regions are denoted by $S_{\epsilon}=(-1,1) \times(-\epsilon / 2, \epsilon / 2)$ and $S_{0}=(-1,1) \times\{0\}$ and their complements, the elastic regions, denoted as $\Omega_{\epsilon}=\Omega \backslash \bar{S}_{\epsilon}$ and $\Omega_{0}=$ $\Omega \backslash S_{0}$.

(2) The sub-spaces of functions on the elastic region which vanish on the top and bottom boundaries are

$$
\begin{aligned}
U & =\left\{u \in H^{1}(\Omega) \mid u(x, \pm 1)=0,-1<x<1\right\}, \\
U_{\epsilon} & =\left\{u \in H^{1}\left(\Omega_{\epsilon}\right) \mid u(x, \pm 1)=0,-1<x<1\right\}, \\
U_{0} & =\left\{u \in H^{1}\left(\Omega_{0}\right) \mid u(x, \pm 1)=0,-1<x<1\right\} .
\end{aligned}
$$

(3) The restriction $\left.u \mapsto u\right|_{\Omega_{\epsilon}}$ is identified as an embedding of the spaces $H^{1}(\Omega) \hookrightarrow$ $H^{1}\left(\Omega_{\epsilon}\right)$ and $U \hookrightarrow U_{\epsilon}$; similarly $H^{1}(\Omega) \hookrightarrow H^{1}\left(\Omega_{0}\right)$ and $U \hookrightarrow U_{0}$.

(4) Below $\chi_{A}$ denotes the characteristic function of $A \subset \Omega ; \chi_{A}(x)=1$ if $x \in A$ and $\chi_{A}(x)=0$ otherwise.

The following lemma quantifies the dependence upon $\epsilon$ of embedding constants and properties of the function spaces for which the energy is continuous and coercive. Here and below $C$ and $c$ denote constants which may vary from instance to instance but will always be independent of $\epsilon$.

Lemma 1.2. Denote the domains and spaces as in Notation 1.1, and if $u^{\epsilon} \in H^{1}\left(\Omega_{\epsilon}\right)$ and $u \in H^{1}\left(\Omega_{0}\right)$ denote by $\left[u^{\epsilon}\right]$ and $[u]$ the jump in their traces across the fault regions;

$$
\left[u^{\epsilon}\right]=u^{\epsilon}(., \epsilon / 2)-u^{\epsilon}(.,-\epsilon / 2) \quad \text { and } \quad[u]=u\left(., 0^{+}\right)-u\left(., 0^{-}\right) .
$$

(1) The constant in Korn's inequality on $U_{\epsilon}$ is independent of $\epsilon$.

(2) The following Poincaré inequality holds for functions in $U$,

$$
(1 / 2)\|u\|_{L^{2}\left(S_{\epsilon}\right)} \leq\left(\epsilon^{2}\left\|u_{y}\right\|_{L^{2}\left(S_{\epsilon}\right)}^{2}+(\epsilon / 2)\|u\|_{H^{1}\left(\Omega_{\epsilon}\right)}^{2}\right)^{1 / 2} .
$$

(3) If $u^{\epsilon} \in H^{1}(\Omega)$ and

$$
u^{\epsilon} \rightarrow u, \quad \chi_{\Omega_{\epsilon}} u_{x}^{\epsilon} \rightarrow g_{0} \quad u_{y}^{\epsilon} \rightarrow g_{1}, \quad \text { in } L^{2}(\Omega),
$$

then $u \in H^{1}(\Omega)$ and $\nabla u=\left(g_{0}, g_{1}\right)^{\top}$.

(4) If $u^{\epsilon} \in H^{1}(\Omega)$ and

$$
u^{\epsilon} \rightarrow u, \quad u_{x}^{\epsilon} \rightarrow g_{0}, \quad \chi_{\Omega_{\epsilon}} u_{y}^{\epsilon} \rightarrow g_{1} \quad \text { in } L^{2}(\Omega),
$$

then $u \in H^{1}\left(\Omega_{0}\right)$ and $\nabla u=\left(g_{0}, g_{1}\right)^{\top}$. In addition $\left[u^{\epsilon}\right] \rightarrow[u]$ in $L^{2}(-1,1)$. 

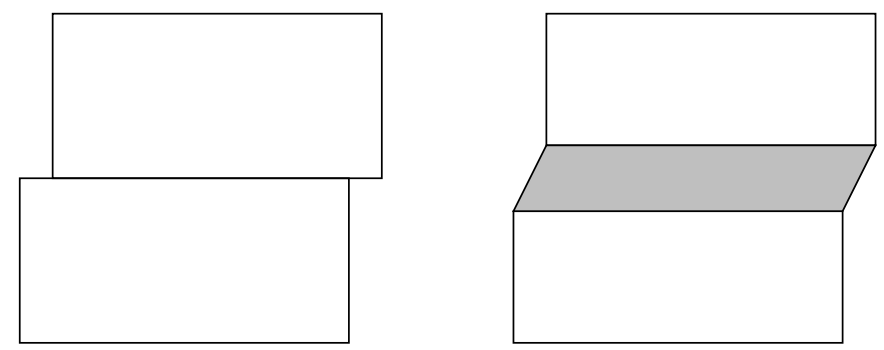

FIG. 3. Approximation of horizontal displacement $u \in U_{0}$ by a function $u^{\epsilon} \in U \subset H^{1}(\Omega)$.

(5) Let $\phi_{\epsilon}: \Omega_{\epsilon} \rightarrow \Omega_{0}$ be the mapping

$\phi_{\epsilon}(x, y)=\left(x, \frac{y-\epsilon / 2}{1-\epsilon / 2}\right), \quad \epsilon / 2<y<1, \quad$ and $\quad \phi_{\epsilon}(x, y)=\left(x, \frac{y+\epsilon / 2}{1-\epsilon / 2}\right), \quad-1<y<-\epsilon / 2$.

Then the linear functions $E_{\epsilon}: H^{1}\left(\Omega_{\epsilon}\right) \rightarrow H^{1}\left(\Omega_{0}\right)$ given by $E_{\epsilon}\left(u^{\epsilon}\right)=u^{\epsilon} \circ \phi_{\epsilon}^{-1}$ are isomorphisms and their norms and the norms of their inverses converge to one as $\epsilon \rightarrow 0$. The restriction of $E_{\epsilon}$ to $U_{\epsilon}$ is an isomorphism onto $U_{0}$.

(6) If $u \in H^{1}\left(\Omega_{0}\right)$, then there exists $u^{\epsilon} \in H^{1}(\Omega)$ such that

(a) $\left\|E_{\epsilon}\left(u^{\epsilon}\right)-u\right\|_{H^{1}\left(\Omega_{0}\right)} \rightarrow 0$.

(b) $\left\|u_{x}^{\epsilon}\right\|_{L^{2}\left(S_{\epsilon}\right)} \rightarrow 0$.

(c) $u_{1 y}^{\epsilon}(x,$.$) is independent of y$ in $S_{\epsilon}$ and $\int_{-\epsilon / 2}^{\epsilon / 2} u_{1 y}^{\epsilon}(., y) d y=\left[u^{\epsilon}\right] \rightarrow[u]$ in $L^{2}(-1,1)$.

In addition, if $u \in U_{0}$, then $u^{\epsilon} \in U$.

The proof of this lemma involves standard arguments [26] so is omitted. The only subtlety appears in the construction of the function $u^{\epsilon}$ in item 6 . The idea is illustrated in Figure 3, given $u \in U_{0}$ extend $u \circ \phi_{\epsilon}^{-1}$ to $S_{\epsilon}$ by linear interpolation. However, to control the $x$-derivative in $S_{\epsilon}$ we first mollify $u$ on each subdomain $\Omega^{ \pm}$with parameter $\sqrt{\epsilon}$.

2. Gamma convergence of the stationary operator. Letting $\mathbb{C}: \mathbb{R}^{2 \times 2} \rightarrow \mathbb{R}_{\text {sym }}^{2 \times 2}$ denote the classical isotropic elasticity tensor with shear and bulk moduli $\mu$ and $\lambda$, the associated strain energy function will be denoted as

$$
2 W(A) \equiv \mathbb{C}(A): A=2 \mu\left(A_{11}^{2}+A_{22}^{2}\right)+\lambda\left(A_{11}+A_{22}\right)^{2}+\mu\left(A_{12}+A_{21}\right)^{2} .
$$

Define $I_{\epsilon}: H^{1}(\Omega)^{2} \rightarrow \mathbb{R}$ to be energy,

$$
\begin{aligned}
I_{\epsilon}(u) & =\int_{\Omega_{\epsilon}} W(\nabla u)+\int_{S_{\epsilon}} W\left(\left[\begin{array}{cc}
u_{1 x} & \sqrt{\epsilon} u_{1 y}-\gamma / \sqrt{\epsilon} \\
\sqrt{\epsilon} u_{2 x} & u_{2 y}
\end{array}\right]\right) \\
& =\int_{\Omega} W\left(\left[\begin{array}{cc}
u_{1 x} & u_{1 y} \chi_{\Omega_{\epsilon}} \\
u_{2 x} \chi_{\Omega_{\epsilon}} & u_{2 y}
\end{array}\right]\right)+\int_{S_{\epsilon}} W\left(\left[\begin{array}{cc}
0 & \sqrt{\epsilon} u_{1 y}-\gamma / \sqrt{\epsilon} \\
\sqrt{\epsilon} u_{2 x} & 0
\end{array}\right]\right) .
\end{aligned}
$$

In this section we establish the following theorem which establishes convergence of the energies in the following sense [11]. 
- (lim-inf inequality) If $\left\{u_{\epsilon}\right\}_{\epsilon>0} \subset U \times U$ with $\left\{I\left(u_{\epsilon}\right)\right\}_{\epsilon>0} \subset \mathbb{R}$ bounded, then there exists $u \in U_{0} \times U$ and a sub-sequence for which $u^{\epsilon} \rightarrow u$ in $H_{l o c}^{1}\left(\Omega_{0}\right) \times H^{1}(\Omega)$ and $I(u) \leq \liminf _{\epsilon \rightarrow 0} I_{\epsilon}\left(u^{\epsilon}\right)$.

- (lim-sup inequality) For each $u \in U_{0} \times U$ there exists a sequence $\left\{u_{\epsilon}\right\}_{\epsilon>0} \subset U \times U$ such that $u^{\epsilon} \rightarrow u$ in $H_{l o c}^{1}\left(\Omega_{0}\right) \times H^{1}(\Omega)$ and $I(u) \geq \lim \sup _{\epsilon \rightarrow 0} I_{\epsilon}\left(u^{\epsilon}\right)$.

Theorem 2.1. Denote the domains and spaces as in Notation 1.1 and let $I_{\epsilon}: U \times U \rightarrow \mathbb{R}$ be as in equation (6) with $\gamma \in L^{2}(-1,1)$ fixed. Assume that the shear and bulk moduli are bounded above, there exists $c_{0}>0$ such that $\mu \geq c_{0}$ and $\mu+\lambda \geq c_{0}$, and that there exists $\epsilon_{0}>0$ such that the shear modulus $\mu$ is independent of $y$ on $S_{\epsilon}$ for $\epsilon<\epsilon_{0}$. Then $I_{\epsilon} \stackrel{\Gamma}{\rightarrow} I$ where $I: U_{0} \times U \rightarrow \mathbb{R}$ is given by

$$
I(u)=\int_{\Omega_{0}} W(\nabla u)+\frac{1}{2} \int_{-1}^{1} \mu\left(\left[u_{1}\right]-\gamma\right)^{2},
$$

for which the strong form of the Euler Lagrange operator is

$-\operatorname{div}(\mathbb{C}(\nabla u))$ on $\Omega_{0}, \quad$ with $\quad[\mathbb{C}(\nabla u)] . n=0$ and $\mathbb{C}(\nabla u)_{12}=\mu\left(\left[u_{1}\right]-\gamma\right)$ on $S_{0}$.

Here [.] denotes the jump across the fault line $y=0$.

The following lemma quantifies the coercivity properties of the energies $I_{\epsilon}$ and the corresponding bounds required for the proof of Theorem 2.1. In this lemma we use the property that in two dimensions the assumptions on the Lame parameters guarantee $W(A) \geq 2 c_{0}\left|A_{\text {sym }}\right|^{2}$ where $A_{\text {sym }}$ denotes the symmetric part of $A \in \mathbb{R}^{2 \times 2}$.

Lemma 2.2. Denote the domains and spaces as in Notation 1.1 and let $I_{\epsilon}: U \times U \rightarrow \mathbb{R}$ be as in equation (6) with $\gamma \in L^{2}(-1,1)$ fixed. Assume that the shear and bulk moduli bounded above, $\mu \geq c_{0}>0$ and $\mu+\lambda \geq c_{0}>0$. Then

$$
\left\|u_{1 x}\right\|_{L^{2}(\Omega)}^{2}+\left\|u_{1 y}\right\|_{L^{2}\left(\Omega_{\epsilon}\right)}^{2}+\left\|u_{2 x}\right\|_{L^{2}\left(\Omega_{\epsilon}\right)}^{2}+\left\|u_{2 y}\right\|_{L^{2}(\Omega)}^{2} \leq C I_{\epsilon}(u),
$$

and

$$
\left\|\sqrt{\epsilon} u_{2 x}\right\|_{L^{2}\left(\Omega_{0}\right)}^{2}+\left\|\sqrt{\epsilon} u_{1 y}\right\|_{L^{2}\left(\Omega_{0}\right)}^{2} \leq C\left(I_{\epsilon}(u)+\|\gamma\|_{L^{2}(-1,1)}^{2}\right) .
$$

In particular,

$$
\left\|u_{1}\right\|_{L^{2}(\Omega)}^{2}+\left\|u_{2}\right\|_{L^{2}(\Omega)}^{2} \leq C\left(I_{\epsilon}(u)+\|\gamma\|_{L^{2}(-1,1)}^{2}\right) .
$$

Proof. It is immediate that

$$
\left\|u_{1 x}\right\|_{L^{2}(\Omega)}^{2}+\left\|u_{1 y}+u_{2 x}\right\|_{L^{2}\left(\Omega_{\epsilon}\right)}^{2}+\left\|u_{2 y}\right\|_{L^{2}(\Omega)}^{2}+\left\|\sqrt{\epsilon}\left(u_{2 x}+u_{1 y}\right)-\gamma / \sqrt{\epsilon}\right\|_{L^{2}\left(S_{\epsilon}\right)}^{2} \leq C I_{\epsilon}(u),
$$

and Korn's inequality on $\Omega_{\epsilon}$ shows

$$
\left\|u_{2 x}\right\|_{L^{2}\left(\Omega_{\epsilon}\right)}^{2}+\left\|u_{1 y}\right\|_{L^{2}\left(\Omega_{\epsilon}\right)}^{2} \leq C\left(\left\|u_{1 y}+u_{2 x}\right\|_{L^{2}\left(\Omega_{\epsilon}\right)}^{2}+\left\|u_{1 x}\right\|_{L^{2}\left(\Omega_{\epsilon}\right)}^{2}+\left\|u_{2 y}\right\|_{L^{2}\left(\Omega_{\epsilon}\right)}^{2}\right) .
$$

Next, use the triangle inequality and the identity $\|\gamma / \sqrt{\epsilon}\|_{L^{2}\left(S_{\epsilon}\right)}=\|\gamma\|_{L^{2}(-1,1)}$ to obtain

$$
\left\|\sqrt{\epsilon}\left(u_{2 x}+u_{1 y}\right)\right\|_{L^{2}\left(S_{\epsilon}\right)} \leq\left\|\sqrt{\epsilon}\left(u_{2 x}+u_{1 y}\right)-\gamma / \sqrt{\epsilon}\right\|_{L^{2}\left(S_{\epsilon}\right)}+\|\gamma\|_{L^{2}(-1,1)},
$$


Korn's inequality for the vector field $\tilde{u}=\sqrt{\epsilon}\left(u_{1}, u_{2}\right)$ on $\Omega_{0}$ shows

$$
\begin{aligned}
& \left\|\sqrt{\epsilon} u_{2 x}\right\|_{L^{2}\left(\Omega_{0}\right)}^{2}+\left\|\sqrt{\epsilon} u_{1 y}\right\|_{L^{2}\left(\Omega_{0}\right)}^{2} \\
& \quad \leq C\left(\left\|\sqrt{\epsilon}\left(u_{2 x}+u_{1 y}\right)\right\|_{L^{2}\left(\Omega_{0}\right)}^{2}+\epsilon\left(\left\|u_{1 x}\right\|_{L^{2}\left(\Omega_{0}\right)}^{2}+\left\|u_{2 y}\right\|_{L^{2}\left(\Omega_{0}\right)}^{2}\right)\right) .
\end{aligned}
$$

Proof. (of Theorem 2.1) Lim-Inf Inequality: Let $\left\{u^{\epsilon}\right\}_{\epsilon>0} \subset H^{1}(\Omega)^{2}$ and suppose $I_{\epsilon}\left(u^{\epsilon}\right)$ is bounded. Lemma 2.2 then shows that the functions

$$
u_{1}^{\epsilon}, u_{1 x}^{\epsilon}, u_{1 y}^{\epsilon} \chi_{\Omega_{\epsilon}}, \quad \text { and } \quad u_{2}^{\epsilon}, u_{2 x}^{\epsilon} \chi_{\Omega_{\epsilon}}, u_{2 y}^{\epsilon},
$$

are all bounded in $L^{2}(\Omega)$. Upon passing to a subsequence we may then assume each of them converges weakly in $L^{2}(\Omega)$ to a limit $u=\left(u_{1}, u_{2}\right) \in L^{2}(\Omega)^{2}$ and from Lemma 1.2 conclude $u \in U_{0} \times U$; in particular,

$$
\left[\begin{array}{cc}
u_{1 x}^{\epsilon} & u_{1 y}^{\epsilon} \chi_{\Omega_{\epsilon}} \\
u_{2 x}^{\epsilon} \chi_{\Omega_{\epsilon}} & u_{2 y}^{\epsilon}
\end{array}\right] \rightarrow\left[\begin{array}{cc}
u_{1 x} & u_{1 y} \\
u_{2 x} & u_{2 y}
\end{array}\right] \quad \text { in } L^{2}(\Omega)^{2 \times 2}
$$

Since $W$ is convex and continuous it is weakly lower semi-continuous; in particular, the limit of the first term in equation (6) is bounded as

$$
\int_{\Omega_{0}} W(\nabla u) \leq \liminf _{\epsilon} \int_{\Omega} W\left(\left[\begin{array}{cc}
u_{1 x}^{\epsilon} & u_{1 y}^{\epsilon} \chi_{\Omega_{\epsilon}} \\
u_{2 x}^{\epsilon} \chi_{\Omega_{\epsilon}} & u_{2 y}^{\epsilon}
\end{array}\right]\right) .
$$

To compute the limit of the second term in equation (6), use Jensen's inequality and the quadratic homogeneity of $W($.$) to obtain$

$$
\begin{aligned}
\int_{S_{\epsilon}} W\left(\left[\begin{array}{cc}
0 & \sqrt{\epsilon} u_{1 y}^{\epsilon}-\gamma / \sqrt{\epsilon} \\
\sqrt{\epsilon} u_{2 x}^{\epsilon} & 0
\end{array}\right]\right) & \geq \int_{-1}^{1} \epsilon W\left(\frac{1}{\epsilon} \int_{-\epsilon / 2}^{\epsilon / 2}\left[\begin{array}{cc}
0 & \left.\left.\sqrt{\epsilon} u_{1 y}^{\epsilon}-\gamma / \sqrt{\epsilon}\right] d y\right) d x \\
\sqrt{\epsilon} u_{2 x}^{\epsilon} & 0
\end{array}\right]\right. \\
& =\int_{-1}^{1} W\left(\left[\begin{array}{cc}
0 & {\left[u_{1}^{\epsilon}\right]-\gamma} \\
\int_{-\epsilon / 2}^{\epsilon / 2} u_{2 x}^{\epsilon} d y & 0
\end{array}\right]\right) d x
\end{aligned}
$$

where $\left[u_{1}^{\epsilon}\right](x)=u_{1}(x, \epsilon / 2)-u_{1}(x,-\epsilon / 2)$. Lemma 1.2 shows $\left[u_{1}^{\epsilon}\right] \rightarrow[u]$ in $L^{2}(-1,1)$, so the lim-inf inequality will follow upon showing that $\int_{-\epsilon / 2}^{\epsilon / 2} u_{2 x}^{\epsilon} d y \rightarrow 0$ in $L^{2}(-1,1)$. To verify this, first use the Cauchy-Schwarz inequality and Lemma 2.2 to bound this term in $L^{2}(-1,1)$,

$$
\int_{-1}^{1}\left(\int_{-\epsilon / 2}^{\epsilon / 2} u_{2 x}^{\epsilon} d y\right)^{2} d x \leq\left\|\sqrt{\epsilon} u_{2 x}^{\epsilon}\right\|_{L^{2}\left(S_{\epsilon}\right)}^{2} \leq C\left(I_{\epsilon}\left(u_{1}^{\epsilon}\right)+\|\gamma\|_{L^{2}(-1,1)}^{2}\right)^{1 / 2} .
$$

To show that this term converges weakly to zero let $\phi \in C_{0}^{\infty}(-1,1)$ and compute

$$
\left|\int_{-1}^{1} \int_{-\epsilon / 2}^{\epsilon / 2} u_{2 x}^{\epsilon} d y \phi d x\right|=\left|\int_{-1}^{1} \int_{-\epsilon / 2}^{\epsilon / 2} u_{2}^{\epsilon} \phi^{\prime} d y d x\right| \leq\left\|u_{2}^{\epsilon}\right\|_{L^{2}\left(S_{\epsilon}\right)} \sqrt{\epsilon}\left\|\phi^{\prime}\right\|_{L^{2}(-1,1)} .
$$

The sharp Poincaré inequality in Lemma 1.2 shows $\left\|u_{2}^{\epsilon}\right\|_{L^{2}\left(S_{\epsilon}\right)} \leq C \sqrt{\epsilon}$ so the right hand side of the above vanishes from which it follows that $\int_{-\epsilon / 2}^{\epsilon / 2} u_{2 x}^{\epsilon} d y \rightarrow 0$ in $L^{2}(-1,1)$.

Lim-Sup Inequality: To construct a recovery sequence for $u \in H^{1}\left(\Omega_{0}\right) \times H^{1}(\Omega)$ select $u^{\epsilon}=\left(u_{1}^{\epsilon}, u_{2}\right)$ where $u_{1}^{\epsilon}$ is the lifting of $u_{1}$ to $H^{1}(\Omega)$ guaranteed by item 6 of Lemma 1.2 . 
Lemma 1.2 shows $u^{\epsilon} \circ \phi_{\epsilon}^{-1} \rightarrow u$ in $H^{1}\left(\Omega_{0}\right)$ where $\phi_{\epsilon}: \Omega_{\epsilon} \rightarrow \Omega_{0}$ is the piecewise affine diffeomorphism in the lemma. Since the mapping $u \mapsto W(\nabla u)$ is continuous on $H^{1}\left(\Omega_{0}\right)^{2}$ it follows that the energy in the bulk converges,

$$
\int_{\Omega_{\epsilon}} W\left(\nabla u^{\epsilon}\right)=\int_{\Omega_{0}} W\left(\nabla\left(u^{\epsilon} \circ \phi_{\epsilon}^{-1}\right)\right)(1-\epsilon / 2) \rightarrow \int_{\Omega_{0}} W(\nabla u) .
$$

The energy in the fault regions $S_{\epsilon}$ takes the form

$$
\int_{S_{\epsilon}} W\left(\left[\begin{array}{cc}
u_{1 x}^{\epsilon} & \sqrt{\epsilon} u_{1 y}^{\epsilon}-\gamma / \sqrt{\epsilon} \\
\sqrt{\epsilon} u_{2 x} & u_{2 y}
\end{array}\right]\right)=\int_{S_{\epsilon}} W\left(\left[\begin{array}{cc}
u_{1 x}^{\epsilon} & \left.\left(\left[u_{1}^{\epsilon}\right]-\gamma\right) / \sqrt{\epsilon}\right] \\
\sqrt{\epsilon} u_{2 x} & u_{2 y}
\end{array}\right]\right) .
$$

Since $u_{2 x}, u_{2 y} \in L^{2}(\Omega)$ are independent of $\epsilon$ and $\left|S_{\epsilon}\right| \rightarrow 0$ it is immediate that $\left\|u_{2 x}\right\|_{L^{2}\left(S_{\epsilon}\right)}$ and $\left\|u_{2 y}\right\|_{L^{2}\left(S_{\epsilon}\right)}$ both converge to zero, and from Lemma 1.2 it follows that $\left\|u_{1 x}^{\epsilon}\right\|_{L^{2}\left(S_{\epsilon}\right)}$ also converges to zero. Also, $\left[u_{1}^{\epsilon}\right]-\gamma$ is independent of $y$ and $\left[u_{1}^{\epsilon}\right] \rightarrow\left[u_{1}\right]$ in $L^{2}(-1,1)$ so

$$
\frac{1}{\sqrt{\epsilon}}\left\|\left[u_{1}^{\epsilon}\right]-\gamma\right\|_{L^{2}\left(S_{\epsilon}\right)}=\left\|\left[u_{1}^{\epsilon}\right]-\gamma\right\|_{L^{2}(-1,1)} \rightarrow\left\|\left[u_{1}\right]-\gamma\right\|_{L^{2}(-1,1)} .
$$

Since $W: \mathbb{R}^{2 \times 2} \rightarrow \mathbb{R}$ is continuous, nonnegative, and has quadratic growth it follows that

$$
\int_{S_{\epsilon}} W\left(\left[\begin{array}{cc}
u_{1 x}^{\epsilon} & \sqrt{\epsilon} u_{1 y}^{\epsilon}-\gamma / \sqrt{\epsilon} \\
\sqrt{\epsilon} u_{2 x} & u_{2 y}
\end{array}\right]\right) \rightarrow \int_{S_{0}} W\left(\left[\begin{array}{cc}
0 & \left(\left[u_{1}\right]-\gamma\right) \\
0 & 0
\end{array}\right]\right)
$$

and $\left\{u^{\epsilon}\right\}_{\epsilon>0} \subset H^{1}(\Omega)^{2}$ is a recovery sequence.

3. Evolution equation. In this section we show that as $\epsilon \rightarrow 0$ solutions of equations (11) -(4) converge to the solution of a limiting problem with the spatial Euler Lagrange operator corresponding to the gamma limit obtained in the previous section.

Solutions of equations (11)-(4) satisfy $(u(t), \gamma(t)) \in U^{2} \times G_{\epsilon}$ and

$$
\begin{aligned}
\int_{\Omega}\left(\rho u_{t t}, \hat{u}\right)+ & \left(\mathbb{C}_{\epsilon}(D(u)), D(\hat{u})\right)+\int_{S_{\epsilon}} \mu\left(\epsilon\left(u_{2 x}+u_{1 y}\right)-\gamma, \hat{u}_{2 x}+\hat{u}_{1 y}\right)=\int_{\Omega}(\rho f, \hat{u}), \\
& \frac{1}{\epsilon} \int_{S_{\epsilon}}(1 / \beta)\left(\gamma_{t}, \hat{\gamma}\right)+\ell(\gamma, \hat{\gamma})-\mu\left(\epsilon\left(u_{2 x}+u_{1 y}\right)-\gamma, \hat{\gamma}\right)=0,
\end{aligned}
$$

for all $(\hat{u}, \hat{\gamma}) \in U^{2} \times G_{\epsilon}$ where

$$
U=\left\{u \in H^{1}(\Omega) \mid u(., \pm 1)=0\right\} \quad \text { and } \quad G_{\epsilon}=\left\{\gamma \in L^{2}\left(S_{\epsilon}\right) \mid \gamma_{x} \in L^{2}\left(S_{\epsilon}\right)\right\} .
$$

In this weak statement $D(u)=(1 / 2)\left(\nabla u+(\nabla u)^{\top}\right)$ is the symmetric part of the displacement gradient and

$$
\mathbb{C}_{\epsilon}(D)=\mathbb{C}\left(\left[\begin{array}{cc}
D_{11} & D_{12} \chi_{\Omega_{\epsilon}} \\
D_{21} \chi_{\Omega_{\epsilon}} & D_{22}
\end{array}\right]\right), \quad \ell(\gamma, \hat{\gamma})=\nu \gamma_{x} \hat{\gamma}_{x}+\hat{\eta} \gamma \hat{\gamma}
$$

where $\mathbb{C}(D)=2 \mu D+\lambda \operatorname{tr}(D) I$ is the isotropic elasticity tensor. 
Solutions of the sharp interface problem satisfy $(u(t), \gamma(t)) \in\left(U_{0} \times U\right) \times G$ and

$$
\begin{gathered}
\int_{\Omega_{0}}\left(\rho u_{t t}, \hat{u}\right)+(\mathbb{C}(D(u)), D(\hat{u}))+\int_{-1}^{1} \mu\left(\left[u_{1}\right]-\gamma,\left[\hat{u}_{1}\right]\right)=\int_{\Omega}(\rho f, \hat{u}), \\
\int_{-1}^{1}(1 / \beta)\left(\gamma_{t}, \hat{\gamma}\right)+\ell(\gamma, \hat{\gamma})-\mu\left(\left[u_{1}\right]-\gamma, \hat{\gamma}\right)=0
\end{gathered}
$$

for all $(\hat{u}, \hat{\gamma}) \in\left(U_{0} \times U\right) \times G$ where

$$
\begin{gathered}
U_{0}=\left\{u \in H^{1}\left(\Omega_{0}\right) \mid u(., \pm 1)=0\right\} \\
U=\left\{u \in H^{1}(\Omega) \mid u(., \pm 1)=0\right\}, \quad \text { and } \quad G=H^{1}(-1,1) .
\end{gathered}
$$

In this section we prove the following theorem which establishes convergence of solutions of equations (7) $-(8)$ to solutions of (9)-(10).

Theorem 3.1. Denote the domains and spaces as in Notation 1.1 and assume that the coefficients in equations (77)-(8) are independent of time and there exist constants $C, c$ such that

$$
0<c \leq \rho(x), \mu(x), \beta(x), \nu(x), \mu(x)+\lambda(x) \leq C, \quad \text { and } \quad 0<\hat{\eta}(x)<C,
$$

and that there exists $\epsilon_{0}>0$ such that the shear modulus $\mu$ is independent of $y$ on $S_{\epsilon}$ for $\epsilon<\epsilon_{0}$.

Fix $f \in L^{1}\left[0, T ; L^{2}(\Omega)\right]$ and initial data $u_{t}(0) \in L^{2}(\Omega), \gamma(0) \in H^{1}(-1,1)$ and $u(0) \in$ $U_{0} \times U$ for the sharp interface problem and let the initial values for equations (77)-(8) be

$$
u_{t}^{\epsilon}(0)=u_{t}(0), \quad u_{2}^{\epsilon}(0)=u_{2}(0), \quad \gamma^{\epsilon}(0)=\gamma(0),
$$

and $u_{1}^{\epsilon}(0)=u_{1}$ if $u_{1}(0) \in U$; otherwise, select $\left\{u_{1}^{\epsilon}(0)\right\}_{\epsilon>0} \subset U$ such that $\left\|u_{1}^{\epsilon}(0)-u_{1}(0)\right\|_{H^{1}\left(\Omega_{\epsilon}\right)} \rightarrow 0$ and $\left\|u_{1 x}^{\epsilon}(0)\right\|_{L^{2}\left(S_{\epsilon}\right)}+\sqrt{\epsilon}\left\|u_{1 y}^{\epsilon}(0)\right\|_{L^{2}\left(S_{\epsilon}\right)} \leq C\left\|u_{1}(0)\right\|_{H^{1}\left(\Omega_{0}\right)}$. Let $\left(u^{\epsilon}, \gamma^{\epsilon}\right)$ denote the solution of (17)-(8) with this data and let $\bar{\gamma}^{\epsilon}(t, x)=(1 / \epsilon) \int_{-\epsilon / 2}^{\epsilon / 2}$ $\gamma^{\epsilon}(t, x, y) d y$. Then $\left\{\left(u^{\epsilon}, \bar{\gamma}^{\epsilon}\right)\right\}_{\epsilon>0}$ converges weakly in $H^{1}\left[0, T ; L^{2}(\Omega)^{2} \times L^{2}(-1,1)\right]$ and strongly in $L^{2}\left[0, T ; L^{2}(\Omega)^{2} \times L^{2}(-1,1)\right]$ to a limit

$$
(u, \gamma) \in \mathcal{U} \equiv H^{1}\left[0, T ; L^{2}(\Omega)^{2} \times L^{2}(-1,1)\right] \cap L^{2}\left[0, T ;\left(U_{0} \times U\right) \times H^{1}(-1,1)\right]
$$

with initial data $(u(0), \gamma(0))$ which satisfies

$$
\begin{gathered}
\int_{0}^{T} \int_{\Omega_{0}}-\left(\rho u_{t}, \hat{u}_{t}\right)+(\mathbb{C}(D(u)), D(\hat{u}))+\int_{0}^{T} \int_{-1}^{1} \mu\left(\left[u_{1}\right]-\bar{\gamma},\left[\hat{u}_{1}\right]\right)=\int_{\Omega}\left(\rho u_{t}(0), \hat{u}(0)\right)+\int_{0}^{T} \int_{\Omega}(\rho f, \hat{u}), \\
\int_{0}^{T} \int_{-1}^{1}(1 / \beta)\left(\gamma_{t}, \hat{\gamma}\right)+\ell(\gamma, \hat{\gamma})-\mu\left(\left[u_{1}\right]-\gamma, \hat{\gamma}\right)=0,
\end{gathered}
$$

for all $(\hat{u}, \hat{\gamma}) \in \mathcal{U}$ with $\hat{u}(T)=0$. 
3.1. Existence of solutions and bounds. Equations (7)-(8) and (9)-(10) both have the structure of a degenerate wave equation on a product space taking the form $(u(t), \gamma(t)) \in$ $\mathbb{U} \times \mathbb{G}$,

$$
C(u, \gamma)_{t t}+B(u, \gamma)_{t}+A(u, \gamma)=(\rho f, 0)
$$

with

$$
C(u, \gamma)=(\rho u, 0), \quad \text { and } \quad B(u, \gamma)=(0, \gamma / \beta),
$$

(the latter scaled by $1 / \epsilon$ for the $\epsilon$ equation) and $A: \mathbb{U} \times \mathbb{G} \rightarrow \mathbb{U}^{\prime} \times \mathbb{G}^{\prime}$ is the Riesz map for the space $\mathbb{U} \times \mathbb{G}$. For the limit problem

$$
A(u, \gamma)(u, \gamma)=\|(u, \gamma)\|_{0}^{2}=\int_{\Omega_{0}} \mathbb{C}(D(u)): D(u)+\int_{-1}^{1} \ell(\gamma, \gamma)+\mu\left(\left[u_{1}\right]-\gamma\right)^{2}
$$

and for the $\epsilon$ equation $A(u, \gamma)(u, \gamma)=\|(u, \gamma)\|_{\epsilon}^{2}$ with

$$
\|(u, \gamma)\|_{\epsilon}^{2}=\int_{\Omega} \mathbb{C}_{\epsilon}(D(u)): D(u)+\int_{S_{\epsilon}}(1 / \epsilon) \ell(\gamma, \gamma)+\mu\left(\sqrt{\epsilon}\left(u_{2 x}+u_{1 y}\right)-\gamma / \sqrt{\epsilon}\right)^{2} .
$$

The hypotheses on the initial data in Theorem 3.1 guarantee $\left\|\left(u^{\epsilon}(0), \gamma^{\epsilon}\right)(0)\right\|_{\epsilon} \rightarrow$ $\|(u(0), \gamma(0))\|_{0}$.

The following theorem from [26, Corollary VI.4.2] establishes existence of (strong) solutions to equations which take the form shown in (11). In the statement of this theorem $\mathcal{L}\left(V, V^{\prime}\right)$ denotes the continuous linear operators and $B \in \mathcal{L}\left(V, V^{\prime}\right)$ is monotone if $B v(v) \geq 0$ for all $v \in V$.

Theorem 3.2. Let $A$ be the Riesz map of the Hilbert space $V$ and let $W$ be the seminormed space obtained from the symmetric and monotone $C \in \mathcal{L}\left(V, V^{\prime}\right)$. Let $D(B) \subset V$ be the domain of a linear monotone operator $B: D(B) \rightarrow V^{\prime}$. Assume that $B+C$ is strictly monotone and $A+B+C: D(B) \rightarrow V^{\prime}$ is surjective. Then for every $f \in$ $C^{1}\left[0, \infty, W^{\prime}\right)$ and every pair $v_{0} \in V$ and $v_{1} \in D(B)$ with $A v_{0}+B v_{1} \in W^{\prime}$, there exists a unique

$$
v \in C[0, \infty, V) \cap C^{1}(0, \infty, V) \cap C^{1}[0, \infty, W) \cap C^{2}(0, \infty, W),
$$

with $v(0)=v_{0}, C v^{\prime}(0)=C v_{1}$ and for each $t>0, v^{\prime} \in D(B), A v(t)+B v^{\prime}(t) \in W^{\prime}$ and

$$
\left(C v^{\prime}(t)\right)^{\prime}+B v^{\prime}(t)+A v(t)=f(t) .
$$

When $V=U^{2} \times G_{\epsilon}$ or $\left(U_{0} \times U\right) \times G$ with operators as in equation (12) the state space is $W=L^{2}(\Omega)$ with weight $\rho$ and $D(B)=V$ is the whole space. Then $(B+C)(u, \gamma)=$ $(\rho u, \gamma / \beta)$ is strictly monotone, and $C+B+A: V \rightarrow V^{\prime}$ is the sum of the Riesz map with a monotone map, so is surjective.

The existence of strong solutions guaranteed by Theorem 3.2 was obtained upon writing equation (13) as a first order system, $\mathcal{B}\left(v, v^{\prime}\right)^{\prime}+\mathcal{A}\left(v, v^{\prime}\right)=\tilde{f}$ with

$$
\mathcal{B}=\left[\begin{array}{cc}
A & 0 \\
0 & C
\end{array}\right], \quad \mathcal{A}=\left[\begin{array}{cc}
0 & -A \\
A & B
\end{array}\right], \quad \text { and } \quad \tilde{f}=\left(\begin{array}{l}
0 \\
f
\end{array}\right) .
$$


Classical semi-group theory then provides necessary and sufficient conditions upon the data for the existence of strong solutions. An alternative to the semi-group approach is to use [27, Proposition III.3.3] which establishes existence of weaker solutions for a broader class of data and problems with time dependent coefficients. Weak solutions exist when $f \in L^{1}\left[0, T ; W^{\prime}\right]$ and satisfy

$$
\left|v^{\prime}\right|_{L^{\infty}[0, T ; W]}^{2}+\|v\|_{L^{\infty}[0, T ; V]}^{2}+\int_{0}^{T} B v^{\prime}\left(v^{\prime}\right) \leq C\left(\left|v^{\prime}(0)\right|_{W}^{2}+\|v(0)\|_{V}^{2}+\|f\|_{L^{1}\left[0, T ; W^{\prime}\right]}^{2}\right) .
$$

The following corollary summarizes bounds available for solutions of (7)-(8) that result from this theory and the Korn and sharp Poincaré inequalities stated in Lemma 1.2.

Corollary 3.3. Under the hypotheses of Theorem 3.1 there exists a constant $C>0$ independent of $\epsilon$ for which solutions $\left(u^{\epsilon}, \gamma^{\epsilon}\right)$ of (7)-(8) satisfy

$$
\begin{aligned}
& \left\|u_{t}^{\epsilon}\right\|_{L^{\infty}\left[0, T ; L^{2}(\Omega)\right]}+\left\|u^{\epsilon}\right\|_{L^{\infty}\left[0, T ; H^{1}\left(\Omega_{\epsilon}\right)\right]} \\
& +(1 / \sqrt{\epsilon})\left\|\gamma_{t}^{\epsilon}\right\|_{L^{2}\left[0, T ; L^{2}\left(S_{\epsilon}\right)\right]}+(1 / \sqrt{\epsilon})\left\|\gamma^{\epsilon}\right\|_{L^{\infty}\left[0, T ; L^{2}\left(S_{\epsilon}\right)\right]}+(1 / \sqrt{\epsilon})\left\|\gamma_{x}^{\epsilon}\right\|_{L^{\infty}\left[0, T ; L^{2}\left(S_{\epsilon}\right)\right]} \\
& +\left\|u_{1 x}\right\|_{L^{\infty}\left[0, T ; L^{2}\left(S_{\epsilon}\right)\right]}+\left\|u_{2 y}\right\|_{L^{\infty}\left[0, T ; L^{2}\left(S_{\epsilon}\right)\right]}+\left\|\sqrt{\epsilon}\left(u_{2 x}^{\epsilon}+u_{1 y}^{\epsilon}\right)-\gamma^{\epsilon} / \sqrt{\epsilon}\right\|_{L^{\infty}\left[0, T ; L^{2}\left(S_{\epsilon}\right)\right]} \\
& \quad \leq C\left(\left\|u_{t}(0)\right\|_{L^{2}(\Omega)}+\|u(0)\|_{H^{1}\left(\Omega_{0}\right)}+\|\gamma(0)\|_{L^{2}(-1,1)}+\|f\|_{L^{1}\left[0, T ; L^{2}(\Omega)\right]}\right) .
\end{aligned}
$$

In particular, $\left\|\mathbb{C}_{\epsilon}\left(\nabla u^{\epsilon}\right)\right\|_{L^{\infty}\left[0, T ; L^{2}(\Omega)^{2 \times 2}\right]}$ and $\left\|\bar{\gamma}_{t}^{\epsilon}\right\|_{L^{2}\left[0, T ; L^{2}(-1,1)\right]}$ and $\left\|\bar{\gamma}^{\epsilon}\right\|_{L^{\infty}\left[0, T ; H^{1}(-1,1)\right]}$ are bounded where $\bar{\gamma}^{\epsilon}(t, x)=(1 / \epsilon) \int_{-\epsilon / 2}^{\epsilon / 2} \gamma^{\epsilon}(t, x, y) d y$ is the average of $\gamma^{\epsilon}$ over the fault region, and the Korn and sharp Poincaré inequalities in Lemma 1.2 imply

$$
\left\|u_{1 y}^{\epsilon}\right\|_{L^{\infty}\left[0, T ; L^{2}\left(S_{\epsilon}\right)\right]}+\left\|u_{2 x}^{\epsilon}\right\|_{L^{\infty}\left[0, T ; L^{2}\left(S_{\epsilon}\right)\right]} \leq C / \sqrt{\epsilon} \quad \text { and } \quad\left\|u^{\epsilon}\right\|_{L^{\infty}\left[0, T ; L^{2}\left(S_{\epsilon}\right)\right]} \leq C \sqrt{\epsilon}
$$

\subsection{Proof of Theorem 3.1. Fix test functions}

$$
\hat{u} \in\left\{\hat{u} \in H^{1}\left[0, T ; H^{2}\left(\Omega_{0}\right) \times H^{2}(\Omega)\right] \mid \hat{u}(., ., \pm 1)=0 \text { and } \hat{u}(T, ., .)=0\right\}
$$

and $\hat{\gamma} \in L^{2}\left[0, T ; H^{1}(-1,1)\right]$, and note that test functions $\hat{u}$ with this regularity are dense in $\left\{\hat{u} \in H^{1}\left[0, T ; U_{0} \times U\right] \mid \hat{u}(T)=0\right\}$. Let $\hat{u}_{1}^{\epsilon} \in H^{1}(\Omega)$ be the function (see Figure 3)

$$
\hat{u}_{1}^{\epsilon}(t, x, y)=\left\{\begin{array}{cc}
\hat{u}_{1}\left(t, x, \frac{y-\epsilon / 2}{1-\epsilon / 2}\right) & \epsilon / 2<y<1, \\
\left(\frac{1}{2}+\frac{y}{\epsilon}\right) \hat{u}_{1}\left(t, x, 0^{+}\right)+\left(\frac{1}{2}-\frac{y}{\epsilon}\right) \hat{u}\left(t, x, 0^{-}\right) & -\epsilon / 2 \leq y \leq \epsilon / 2, \\
\hat{u}_{1}\left(t, x, \frac{y+\epsilon / 2}{1-\epsilon / 2}\right) & -1<y<-\epsilon / 2,
\end{array}\right.
$$

and set the test functions in equations (7)-(8) to be $\hat{u}^{\epsilon}=\left(\hat{u}_{1}^{\epsilon}, \hat{u}_{2}\right) \in U_{\epsilon}$ and $\hat{\gamma}(t, x, y)=$ $\hat{\gamma}(t, x)$ and integrate the equation for $u^{\epsilon}$ by parts in time to get

$$
\begin{aligned}
& \int_{0}^{T} \int_{\Omega}-\left(\rho u_{t}^{\epsilon}, \hat{u}_{t}^{\epsilon}\right)+\left(\mathbb{C}_{\epsilon}\left(D\left(u^{\epsilon}\right)\right), D\left(\hat{u}^{\epsilon}\right)\right)+\int_{0}^{T} \int_{-1}^{1} \mu\left(\left[u_{1}^{\epsilon}\right]-\bar{\gamma}^{\epsilon},\left[\hat{u}_{1}\right]\right) \\
+ & \int_{0}^{T} \int_{S_{\epsilon}} \mu\left(\epsilon\left(u_{2 x}^{\epsilon}+u_{1 y}^{\epsilon}\right)-\gamma^{\epsilon}, \hat{u}_{2 x}\right)+\mu\left(u_{2 x}^{\epsilon},\left[\hat{u}_{1}\right]\right)=\int_{\Omega}\left(\rho u_{t}^{\epsilon}(0), \hat{u}^{\epsilon}(0)\right)+\int_{0}^{T} \int_{\Omega}\left(f, \hat{u}^{\epsilon}\right),
\end{aligned}
$$


and

$$
\int_{0}^{T} \int_{-1}^{1}(1 / \beta)\left(\bar{\gamma}_{t}^{\epsilon}, \hat{\gamma}\right)+\ell\left(\bar{\gamma}^{\epsilon}, \hat{\gamma}\right)-\mu\left(\left[u_{1}^{\epsilon}\right]-\bar{\gamma}^{\epsilon}, \hat{\gamma}\right)-\int_{0}^{T} \int_{S_{\epsilon}} \mu\left(u_{2 x}^{\epsilon}, \hat{\gamma}\right)=0
$$

where $\bar{\gamma}^{\epsilon}(t, x)=(1 / \epsilon) \int_{-\epsilon / 2}^{\epsilon / 2} \gamma^{\epsilon}(t, x, y) d y$ is the average shear in the fault region. The last terms on the left of these two equations represent the "consistency error" corresponding to approximating a fault region of finite width with a sharp interface. We verify that these terms vanish as $\epsilon \rightarrow 0$, and upon passing to a sub-sequence the remaining terms consist of weakly converging terms paired with a strongly converging test function, so the limits of these pairings are the pairings of their limits from which the theorem follows.

Using the bounds in Corollary 3.3 and Lemma 1.2 we may pass to a sub-sequence for which

$$
\begin{array}{cll}
u_{t}^{\epsilon} \rightarrow^{*} u_{t}, & \text { in } & L^{\infty}\left[0, T ; L^{2}(\Omega)^{2}\right], \\
\mathbb{C}_{\epsilon}\left(D u^{\epsilon}\right) \rightarrow^{*} \mathbb{C}(D u), & \text { in } \quad L^{\infty}\left[0, T ; L^{2}(\Omega)^{2 \times 2}\right], \\
{\left[u_{1}^{\epsilon}\right] \rightarrow^{*}\left[u_{1}\right],} & \text { in } L^{\infty}\left[0, T ; L^{2}(-1,1)\right], \\
\bar{\gamma}^{\epsilon} \rightarrow^{*} \gamma, & \text { in } L^{\infty}\left[0, T ; L^{2}(-1,1)\right], \\
\bar{\gamma}_{t}^{\epsilon} \gamma_{t}, & \text { in } L^{2}\left[0, T ; L^{2}(-1,1)\right], \\
\bar{\gamma}_{x}^{\epsilon} \rightarrow^{*} \gamma_{x}, & \text { in } L^{\infty}\left[0, T ; L^{2}(-1,1)\right] .
\end{array}
$$

The first two terms in equations (14) and (15) are paired with the test functions

$$
\begin{array}{cc}
\hat{u}_{t}^{\epsilon} \rightarrow \hat{u}_{t}, & \text { in } \quad L^{1}\left[0, T ; L^{2}(\Omega)^{2}\right], \\
{\left[\begin{array}{cc}
\hat{u}_{1 x}^{\epsilon} & \hat{u}_{1 y}^{\epsilon} \chi_{\Omega_{\epsilon}} \\
\hat{u}_{2 x} \chi_{\Omega_{\epsilon}} & \hat{u}_{2 y}^{\epsilon}
\end{array}\right] \rightarrow \nabla \hat{u},} & \text { in } \quad L^{\infty}\left[0, T ; L^{2}\left(\Omega_{0}\right)^{2 \times 2}\right],
\end{array}
$$

and the terms involving $\left[u_{1}^{\epsilon}\right]-\bar{\gamma}^{\epsilon}$ are paired with test functions independent of $\epsilon$, from which it follows that the first three terms on the left hand side of equations (14) and (15) converge as claimed.

The Cauchy-Schwarz inequality and the smoothness of the test function $\hat{u}_{2}(t) \in$ $H^{2}(\Omega) \hookrightarrow W^{1,4}(\Omega)$ are used to estimate the first consistency error term in equation (14),

$$
\begin{aligned}
& \int_{0}^{T} \int_{S_{\epsilon}} \mu\left(\epsilon\left(u_{2 x}^{\epsilon}+u_{1 y}^{\epsilon}\right)-\bar{\gamma}^{\epsilon}, \hat{u}_{2 x}\right) \\
& \quad \leq C\left\|\sqrt{\epsilon}\left(u_{2 x}^{\epsilon}+u_{1 y}^{\epsilon}\right)-\gamma^{\epsilon} / \sqrt{\epsilon}\right\|_{L^{\infty}\left[0, T ; L^{2}\left(S_{\epsilon}\right)\right]}\left\|\hat{u}_{2 x}\right\|_{L^{1}\left[0, T ; L^{2}\left(S_{\epsilon}\right)\right]} \\
& \quad \leq C\left\|\sqrt{\epsilon}\left(u_{2 x}^{\epsilon}+u_{1 y}^{\epsilon}\right)-\gamma^{\epsilon} / \sqrt{\epsilon}\right\|_{L^{\infty}\left[0, T ; L^{2}\left(S_{\epsilon}\right)\right]}\left\|\hat{u}_{2 x}\right\|_{L^{1}\left[0, T ; L^{4}\left(S_{\epsilon}\right)\right]} \epsilon^{1 / 4} \\
& \quad \rightarrow 0
\end{aligned}
$$

The final terms on the left hand side of equations (14) and (15) involve $u_{2 x}^{\epsilon}$ paired with test functions which are independent of $y$. It then suffices to show that $\bar{u}_{2 x}^{\epsilon} \equiv$ $\int_{-\epsilon / 2}^{\epsilon / 2} u_{2 x}^{\epsilon}(., ., y) d y$ converges weakly star to zero in $L^{\infty}\left[0, T ; L^{2}(-1,1)\right]$. To do this the 
Cauchy-Schwarz inequality and Corollary 3.3 are used to first show that it is bounded,

$$
\int_{-1}^{1}\left(\int_{-\epsilon / 2}^{\epsilon / 2} u_{2 x}^{\epsilon}(t, x, y) d y\right)^{2} d x \leq \int_{-1}^{1} \epsilon \int_{-\epsilon / 2}^{\epsilon / 2} u_{2 x}^{\epsilon}(t, x, y)^{2} d y d x=\epsilon\left\|u_{2 x}^{\epsilon}(t)\right\|_{L^{2}\left(S_{\epsilon}\right)}^{2} \leq C .
$$

To establish weak star convergence to zero it then suffices to test against smooth functions $\hat{\phi}$ with compact support in $(0, T) \times \Omega$ since they are dense in $L^{1}\left[0, T ; L^{2}(-1,1)\right]$,

$\int_{0}^{T} \int_{-1}^{1} \int_{-\epsilon / 2}^{\epsilon / 2}\left(\hat{u}_{2 x}^{\epsilon}, \hat{\phi}\right)=\int_{0}^{T} \int_{-1}^{1} \int_{-\epsilon / 2}^{\epsilon / 2}-\left(\hat{u}_{2}^{\epsilon}, \phi_{x}\right) \leq\left\|\hat{u}_{2}^{\epsilon}\right\|_{L^{\infty}\left[0, T ; L^{2}\left(S_{\epsilon}\right)\right]}\|\hat{\phi}\|_{L^{1}\left[0, T ; L^{2}\left(S_{\epsilon}\right)\right]} \leq C \sqrt{\epsilon}$.

It follows that the limit $(u, \gamma)$ is a solution of the sharp interface problem, and the theorem follows provided it takes the specified initial values. However, this is direct since $\left(u^{\epsilon}, \bar{\gamma}^{\epsilon}\right)$ converges weakly in $H^{1}\left[0, T ; L^{2}(\Omega)^{2} \times L^{2}(-1,1)\right]$ from which it follows that the initial values of the limit $(u, \gamma)$ are the limit of the initial values.

4. Numerical examples. This section first presents a numerical example to exhibit the contrast between direct numerical simulation of the stationary form of equations (1) -(4) and the limit problem considered in Section 2. In the second section numerical approximation of a singular solution corresponding to a dislocation is presented.

In the numerical examples below the parameters are set to

$$
\mu=1, \quad \lambda=2, \quad a=1 / 2, \quad \ell=1 / 4, \quad \hat{\eta}=2, \quad \nu=0, \quad \epsilon=1 / 10,
$$

and for the limit problem uniform rectangular elements of size $h=1 / n$ with $n \in \mathbb{N}$ are utilized. When $\epsilon>0$ the fault region is meshed with rectangular elements of size $1 / n \times \epsilon / n$; the mesh with $n=4$ is illustrated in Figure 1) Galerkin approximations of the solution to the elasticity problems are computed using the piecewise quadratic finite element spaces on these meshes.

4.1. Classical solution. A piecewise smooth solution of the limit problem with constant Lame parameters is constructed by setting

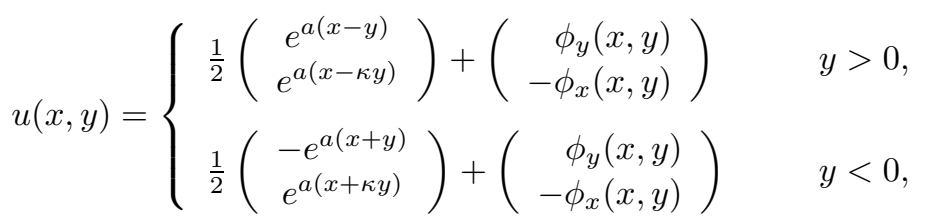

where $\kappa=\lambda /(2 \mu+\lambda)$ and $\phi(x, y)=e^{-\ell y} \cos (\ell x)$. Then

$$
\left[u_{1}(x)\right]=e^{a x}, \quad \gamma(x) \equiv\left[u_{1}\right]-(1 / \mu) \mathbb{C}(\nabla u)_{12}=e^{a x}-2 \ell^{2} \cos (\ell x),
$$

and right hand sides for the stationary problem are manufactured so that the equations are satisfied,

$$
f=-\operatorname{div}(\mathbb{C}(\nabla u)) \quad \text { and } \quad f_{0}=\hat{\eta} \gamma-\mathbb{C}(\nabla u)_{12}-\nu \gamma_{x x} .
$$


To exhibit the differences between direct numerical approximation of (11)-(4) and numerical approximation of the limiting problem we first tabulate the errors, $u^{0}-u_{h}^{0}$, of the numerical approximation of the solution (16). Numerical approximations $u_{h}^{\epsilon}$ of the stationary equations (1)-(4) are then computed using the same boundary data and body force $f$. While the exact solution, $u^{\epsilon}$, of the problem with this data is not known, we tabulate (norms of) the differences $u^{0}-u_{h}^{\epsilon}$ for $\epsilon$ fixed. As $h \rightarrow 0$ this difference converges to the "modeling" error $u^{0}-u^{\epsilon}$ associated with approximating the fault region by a surface. An estimate of the mesh size required to resolve the deformation in the fault region is obtained by observing when difference $u^{0}-u_{h}^{\epsilon}$ stabilizes. Note that in general $\lim _{\epsilon \rightarrow}\left\|u^{0}-u^{\epsilon}\right\|_{L^{2}(\Omega)} \rightarrow 0$ but $\lim _{\epsilon \rightarrow}\left\|u^{0}-u^{\epsilon}\right\|_{H^{1}\left(\Omega_{0}\right)} \not \rightarrow 0$.

4.1.1. Uncoupled problem. Table 1 exhibits the errors in the numerical approximation of the solution (16) of the limit problem considered in Section 2 with $\gamma$ the function specified in (17). The optimal third order rate in $L^{2}(\Omega)$ and second order rate for the derivatives is clear. Norms of the differences $u^{0}-u_{h}^{\epsilon}$ are presented in Table 2, For this example it is clear that very accurate solutions of the limit problem can be computed on very modest meshes while resolution of the deformation in the fault region requires significantly finer meshes. The norms computed on the finest meshes give an estimate of the modeling error $\left\|u^{0}-u^{\epsilon}\right\|_{L^{2}(\Omega)} \simeq 0.34$. Representative solutions for each of the problems are illustrated in Figures 4 and 5

TABLE 1. Errors for the uncoupled limit problem $(\epsilon=0)$.

\begin{tabular}{|c|c|c|r|}
\hline$h$ & $\left\|u^{0}-u_{h}^{0}\right\|_{L^{2}(\Omega)}$ & $\left\|u^{0}-u_{h}^{0}\right\|_{H^{1}\left(\Omega_{0}\right)}$ & \# unknowns \\
\hline $1 / 8$ & $1.684735 \mathrm{e}-05$ & $4.402589 \mathrm{e}-04$ & 629 \\
$1 / 16$ & $2.098734 \mathrm{e}-06$ & $1.091350 \mathrm{e}-04$ & 2277 \\
$1 / 32$ & $2.617986 \mathrm{e}-07$ & $2.718018 \mathrm{e}-05$ & 8645 \\
$1 / 64$ & $3.268957 \mathrm{e}-08$ & $6.783206 \mathrm{e}-06$ & 33669 \\
$1 / 128$ & $4.084190 \mathrm{e}-09$ & $1.694404 \mathrm{e}-06$ & 132869 \\
$1 / 256$ & $5.154866 \mathrm{e}-10$ & $4.234319 \mathrm{e}-07$ & 527877 \\
\hline Norms & 1.435134 & 1.662724 & \\
\hline
\end{tabular}

TABLE 2. Differences between the uncoupled $\epsilon$-problem and limit problem with $\epsilon=0.1$.

\begin{tabular}{|c|c|c|r|}
\hline$h$ & $\left\|u^{0}-u_{h}^{\epsilon}\right\|_{L^{2}(\Omega)}$ & $\left\|u^{0}-u_{h}^{\epsilon}\right\|_{H^{1}\left(\Omega_{0}\right)}$ & \# unknowns \\
\hline $1 / 8$ & $2.148338 \mathrm{e}-01$ & $4.553696 \mathrm{e}+00$ & 1666 \\
$1 / 16$ & $3.029500 \mathrm{e}-01$ & $5.114175 \mathrm{e}+00$ & 6402 \\
$1 / 32$ & $3.183221 \mathrm{e}-01$ & $5.119497 \mathrm{e}+00$ & 25090 \\
$1 / 64$ & $3.358588 \mathrm{e}-01$ & $5.177173 \mathrm{e}+00$ & 99330 \\
$1 / 128$ & $3.459277 \mathrm{e}-01$ & $5.223399 \mathrm{e}+00$ & 395266 \\
$1 / 256$ & $3.433117 \mathrm{e}-01$ & $5.218226 \mathrm{e}+00$ & 1576962 \\
\hline
\end{tabular}




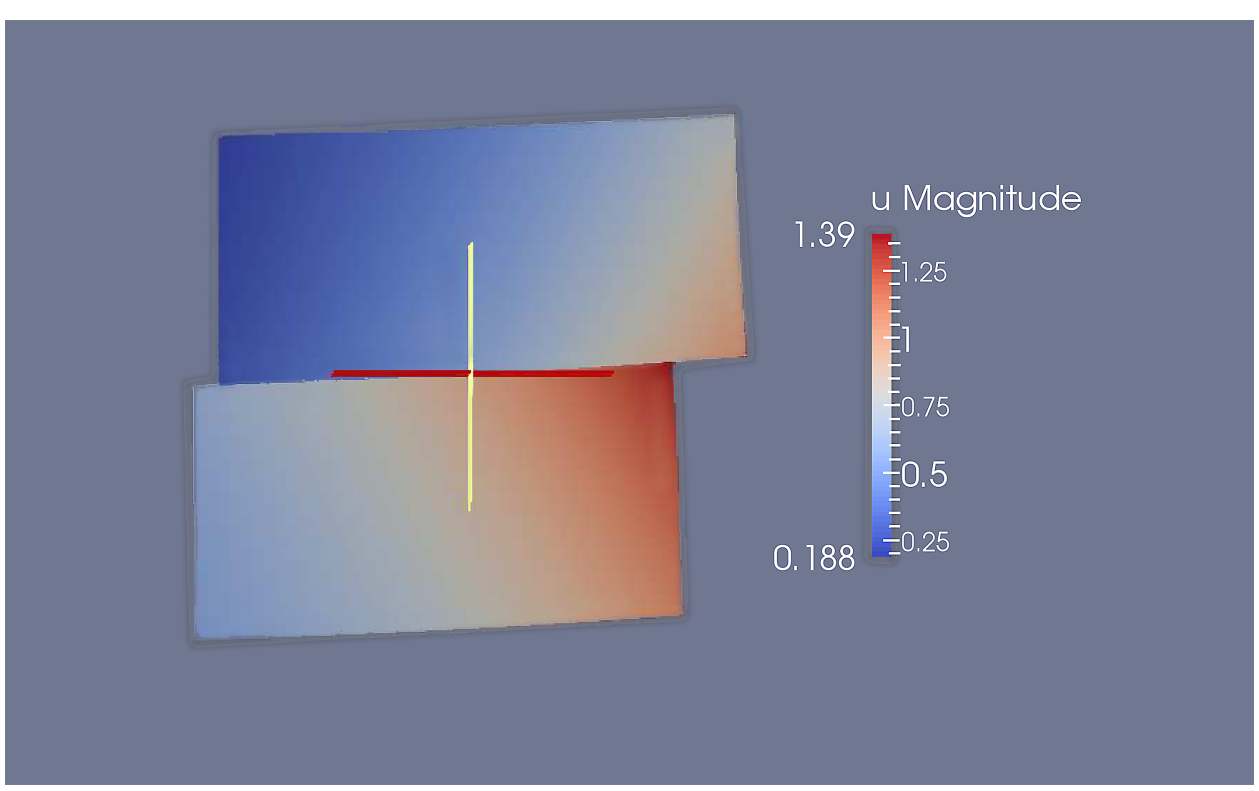

FIG. 4. Solution of the uncoupled limit problem mesh size $h=1 / 256$.

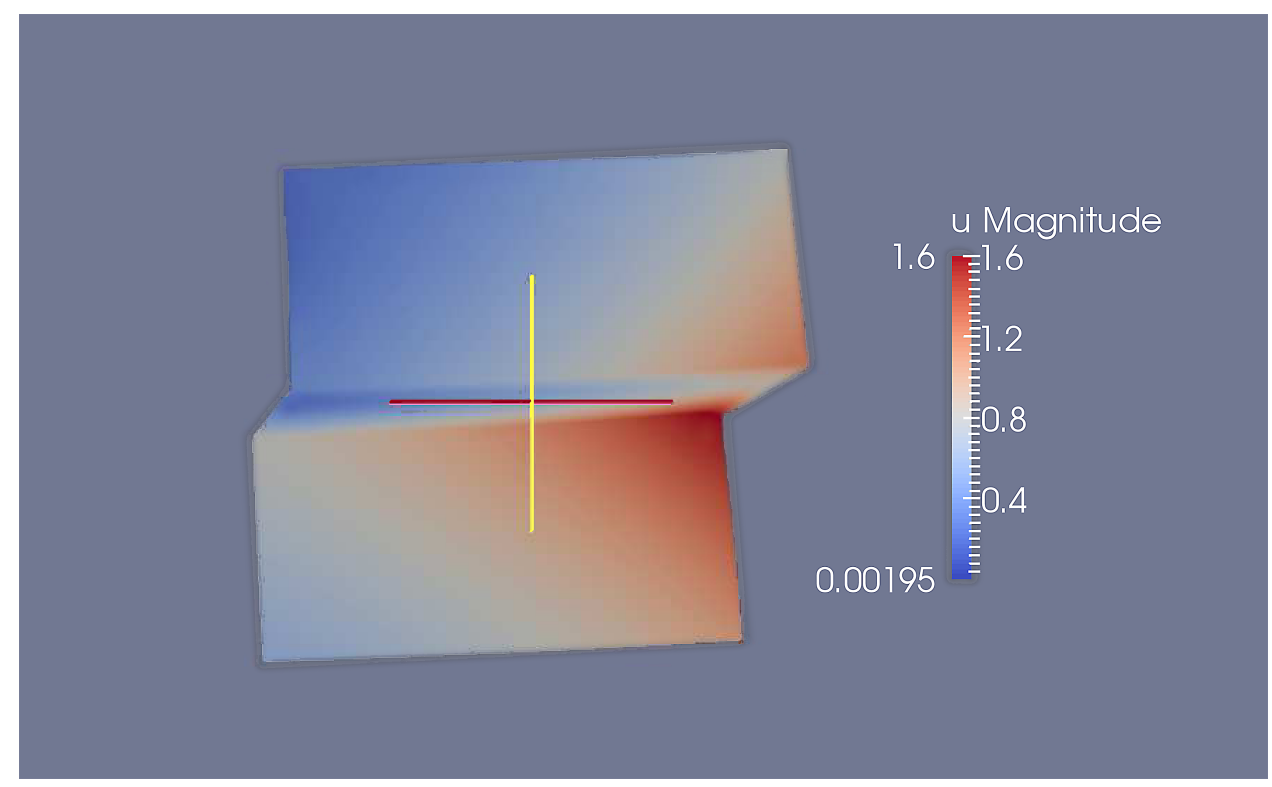

FIG. 5. Solution of the uncoupled problem with $\epsilon=0.1, h=1 / 256$. 
4.1.2. Coupled problems. Table 3 exhibits the errors for the coupled problem when numerical approximations of both $u$ and $\gamma$ are computed using the limit energy given in equation (5). Again the optimal third order rate in the $L^{2}$ norms for both $u$ and $\gamma$ and second order rate for the derivatives of $u$ is obtained. Norms of the differences $u^{0}-u_{h}^{\epsilon}$ and $\gamma-\gamma_{h}^{\epsilon}$ are presented in Table 4. As for the uncoupled case, very accurate solutions of the limit problem can be computed on modest meshes while resolution of the deformation in the fault region requires finer meshes. The modeling errors for this problem are $\left\|u^{0}-u^{\epsilon}\right\|_{L^{2}(\Omega)} \simeq 0.16$ and $\left\|\gamma-\gamma^{\epsilon}\right\| \simeq 0.082$. Representative deformations are illustrated in Figures 6 and 7 .

TABLE 3. Errors for the coupled limit problem $(\epsilon=0)$.

\begin{tabular}{|c|c|c|c|r|}
\hline$h$ & $\left\|u^{0}-u_{h}^{0}\right\|_{L^{2}(\Omega)}$ & $\left\|u^{0}-u_{h}^{0}\right\|_{H^{1}\left(\Omega_{0}\right)}$ & $\left\|\gamma-\gamma_{h}\right\|_{L^{2}(-1,1)}$ & $\#$ unknowns \\
\hline $1 / 8$ & $1.683317 \mathrm{e}-05$ & $4.403035 \mathrm{e}-04$ & $1.597969 \mathrm{e}-05$ & 646 \\
$1 / 16$ & $2.098047 \mathrm{e}-06$ & $1.091376 \mathrm{e}-04$ & $2.077018 \mathrm{e}-06$ & 2310 \\
$1 / 32$ & $2.617686 \mathrm{e}-07$ & $2.718031 \mathrm{e}-05$ & $2.646097 \mathrm{e}-07$ & 8710 \\
$1 / 64$ & $3.268836 \mathrm{e}-08$ & $6.783211 \mathrm{e}-06$ & $3.338419 \mathrm{e}-08$ & 33798 \\
$1 / 128$ & $4.084169 \mathrm{e}-09$ & $1.694404 \mathrm{e}-06$ & $4.191933 \mathrm{e}-09$ & 133126 \\
$1 / 256$ & $5.161811 \mathrm{e}-10$ & $4.234319 \mathrm{e}-07$ & $5.258066 \mathrm{e}-10$ & 528390 \\
\hline Norms & 1.435134 & 1.662724 & 1.365834 & \\
\hline
\end{tabular}

TABle 4. Differences between coupled $\epsilon$-problem and limit problem with $\epsilon=0.1$.

\begin{tabular}{|c|c|c|c|r|}
\hline$h$ & $\left\|u^{0}-u_{h}^{\epsilon}\right\|_{L^{2}(\Omega)}$ & $\left\|u^{0}-u_{h}^{\epsilon}\right\|_{H^{1}\left(\Omega_{0}\right)}$ & $\left\|\gamma-\gamma_{h}^{\epsilon}\right\|_{L^{2}\left(S_{\epsilon}\right)}$ & \# unknowns \\
\hline $1 / 8$ & $1.575165 \mathrm{e}-01$ & $4.626232 \mathrm{e}+00$ & $4.662827 \mathrm{e}-02$ & 1955 \\
$1 / 16$ & $1.582971 \mathrm{e}-01$ & $4.628384 \mathrm{e}+00$ & $5.548420 \mathrm{e}-02$ & 7491 \\
$1 / 32$ & $1.584715 \mathrm{e}-01$ & $4.655150 \mathrm{e}+00$ & $6.875543 \mathrm{e}-02$ & 29315 \\
$1 / 64$ & $1.594472 \mathrm{e}-01$ & $4.670685 \mathrm{e}+00$ & $7.885558 \mathrm{e}-02$ & 115971 \\
$1 / 128$ & $1.596179 \mathrm{e}-01$ & $4.675027 \mathrm{e}+00$ & $8.156652 \mathrm{e}-02$ & 461315 \\
$1 / 256$ & $1.593571 \mathrm{e}-01$ & $4.676821 \mathrm{e}+00$ & $8.152622 \mathrm{e}-02$ & 1840131 \\
\hline
\end{tabular}




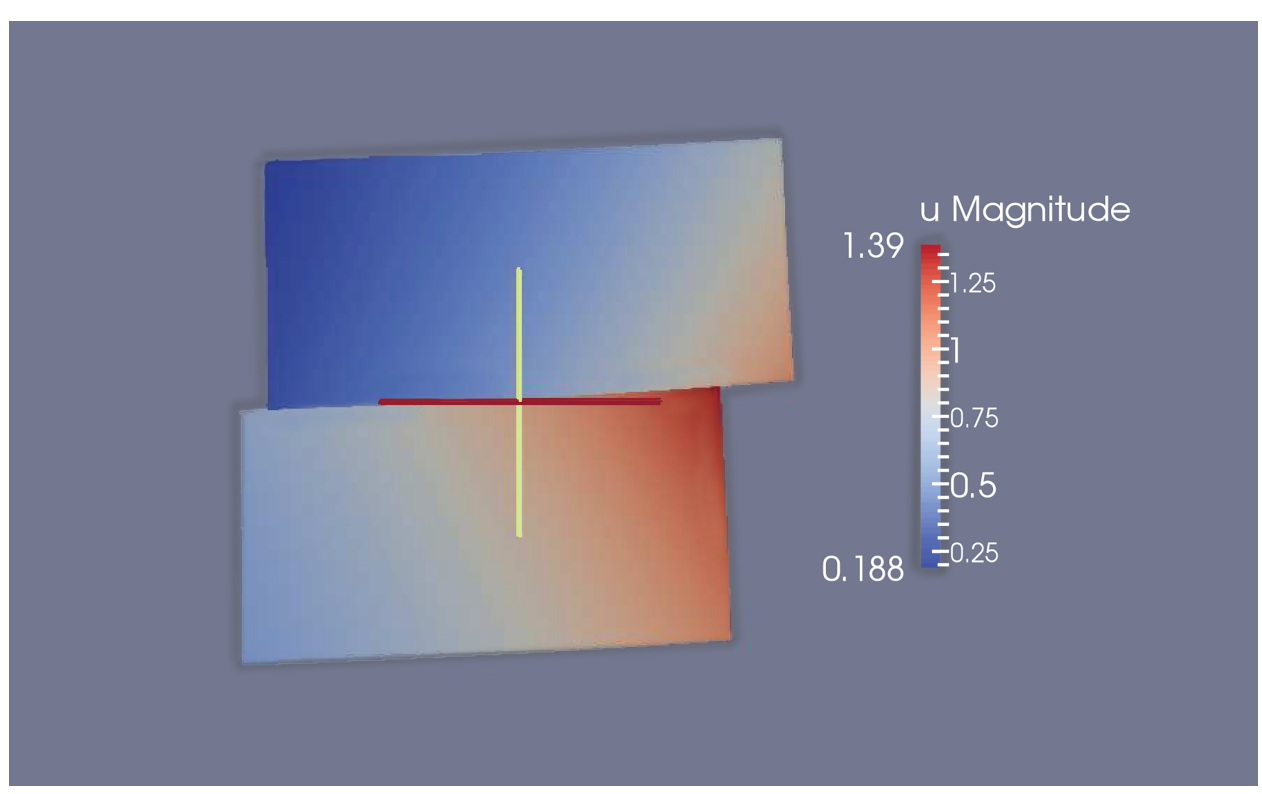

FIG. 6. Displacement of coupled limit problem with $h=1 / 256$.

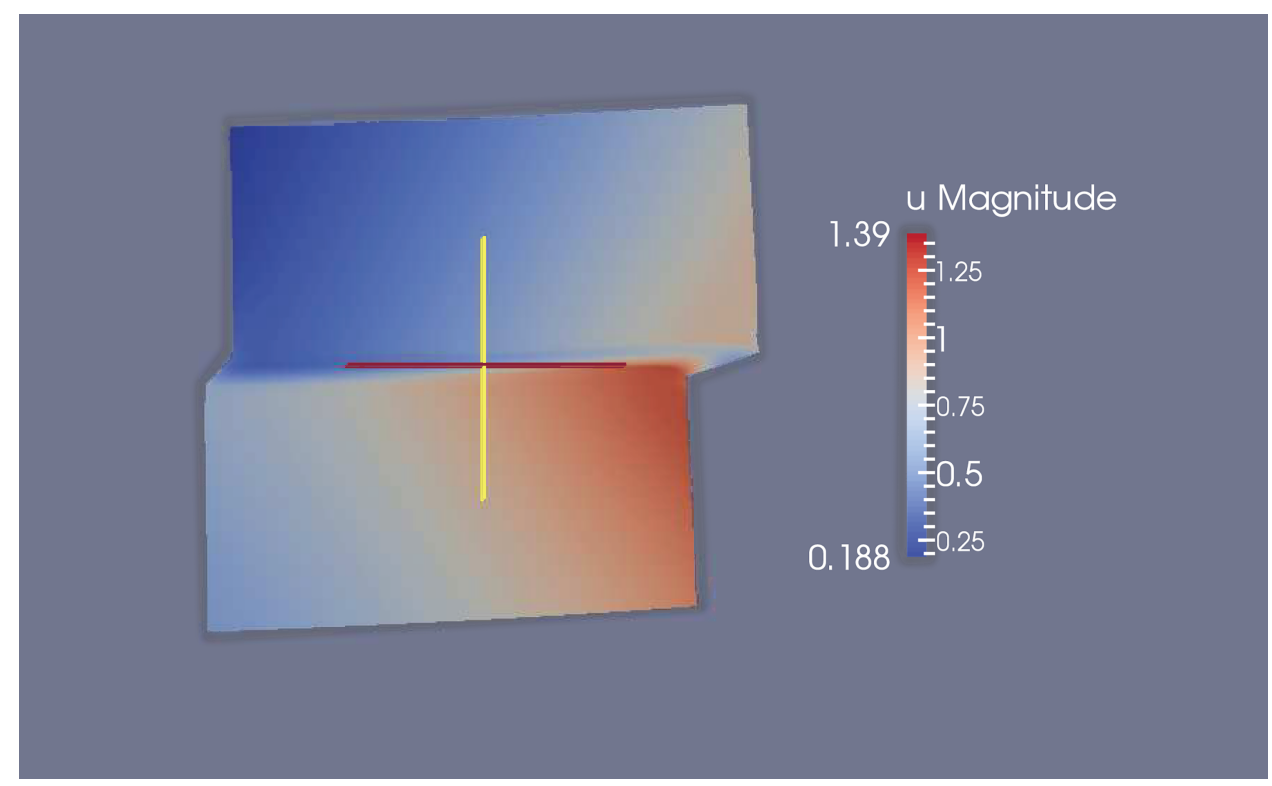

FIG. 7. Displacement for coupled problem with $\epsilon=0.1$ and $h=1 / 256$. 
4.2. Dislocation. An explicit solution for the solution of the linear elasticity problem with an edge dislocation along the $z$-axis is 21]

$u(x, y)=\frac{1}{2 \pi}\left(\begin{array}{c}\arctan (y / x)+\frac{x y}{2(1-\hat{\nu})\left(x^{2}+y^{2}\right)} \\ \frac{-1}{4(1-\hat{\nu})}\left((1-2 \hat{\nu}) \ln \left(x^{2}+y^{2}\right)+\frac{x^{2}-y^{2}}{x^{2}+y^{2}}\right)\end{array}\right)$, with Poisson ratio $\hat{\nu}=\frac{\lambda}{2(\mu+\lambda)}$

This solution, illustrated in Figure 8, represents the displacement that results when a dislocation, currently at the origin, has propagated along the negative $x$-axis so that $\left[u_{1}(x)\right]=1$ for $x<0$ and $\left[u_{1}(x)\right]=0$ for $x>0$. The stress has a singularity of order $O(1 / r)$ at the origin and is otherwise continuous, and the displacement is square integrable but its derivatives are not. While the results of the prior sections are not applicable to singular solutions, almost singular solutions arise in engineering practice so it is important for the numerical schemes to be robust in this context.
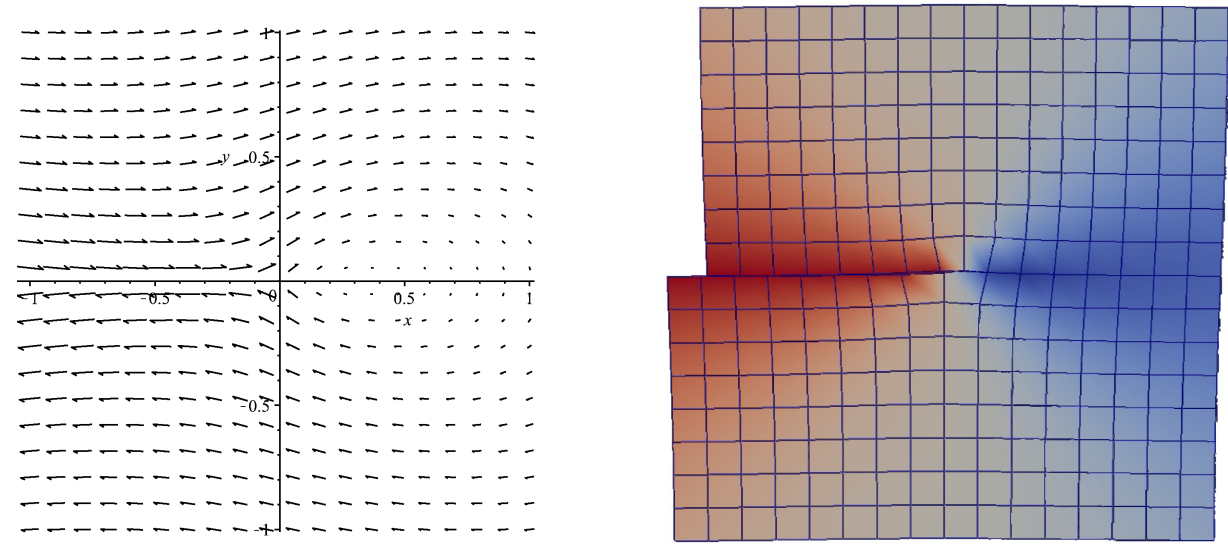

FIG. 8. Displacement field with dislocation at the origin and numerical approximation.

To illustrate the robustness properties of codes using the limit energy a singular solution of the stationary limit problem is manufactured by setting $\gamma=\left[u_{1}\right]-(1 / \mu) T_{12}$, so that the jump condition is satisfied, and the nonhomogeneous right hand side for the equation for $\gamma$,

$$
f_{0}(x)=\hat{\eta} \gamma(x)-T_{12}(x, 0)=\hat{\eta}\left[u_{1}(x)\right]+\frac{(\lambda+\mu)(\hat{\eta}+\mu)}{\pi(2 \mu+\lambda) x} .
$$

(Since $\gamma_{x x}$ does not exist we set the coefficient of this term to be zero.) Inner products of this (nonintegrable) function with basis functions were approximated using Gaussian quadrature. Table 5 shows that the error $\left\|u-u_{h}\right\|_{L^{2}(\Omega)}$ converges linearly with $h$ and $\left\|\gamma_{h}\right\|_{L^{2}(\Omega)} \simeq O(1 / \sqrt{h})$ diverges since the limit $\gamma(x) \simeq O(1 / x)$ is not integrable. Figure 8 illustrates the deformation computed with quadratic elements on a $16 \times 16$ grid. 
TABLE 5. Errors in displacement and norm of shear for dislocation example.

\begin{tabular}{|c|c|c|}
\hline$h$ & $\left\|u-u_{h}\right\|_{L^{2}(\Omega)}$ & $\left\|\gamma_{h}\right\|_{L^{2}(-1,1)}$ \\
\hline $1 / 8$ & $1.226581 \mathrm{e}-02$ & 7.005017 \\
$1 / 16$ & $6.141691 \mathrm{e}-03$ & 9.835010 \\
$1 / 32$ & $3.073164 \mathrm{e}-03$ & 13.85832 \\
$1 / 64$ & $1.537180 \mathrm{e}-03$ & 19.56296 \\
$1 / 128$ & $7.687419 \mathrm{e}-04$ & 27.64101 \\
$1 / 256$ & $3.844092 \mathrm{e}-04$ & 39.07248 \\
\hline
\end{tabular}

Appendix A. Derivation from a plasticity model. Displacements and gradients are assumed to be small in the region $\Omega_{\epsilon}=\Omega \backslash \bar{S}_{\epsilon}$ outside the fault so that the motion is governed by the equations of linear elasticity,

$$
\rho u_{t t}-\operatorname{div}(\mathbb{C}(\nabla u))=\rho f, \quad \text { in } \Omega_{\epsilon} .
$$

Small displacement plasticity theory models the motion in the fault region $S_{\epsilon}$. In this theory the elastic deformation tensor, $U$, deviates from $\nabla u$ due to slips and motion of defects. The balance of linear momentum becomes

$$
\rho u_{t t}-\operatorname{div}(\mathbb{C}(U))=\rho f, \quad \text { in } S_{\epsilon},
$$

and evolution of $U$ is governed by an equation of the form

$$
U_{t}-\nabla u_{t}+\operatorname{Curl}(U) \times v_{d}=0,
$$

where $v_{d}$ is a constitutively specified defect velocity. In this equation the $\operatorname{Curl}($.$) and$ cross product of a matrix act row-wise;

$$
\operatorname{Curl}(U)_{m n}=\epsilon_{i j n} U_{m j, i}, \quad \text { and } \quad(A \times v)_{m n}=\epsilon_{i j n} A_{m i} v_{j} .
$$

The defect velocity $v_{d}$ is chosen to model the (typically large) dissipation due to defect motion, and local energy changes due to distortion in the material during passage of a defect. In the following lemma the axial vector of the skew part of a matrix $A$ is denoted by $X(A)$; that is,

$$
X(A)_{i}=\epsilon_{i j k} A_{j k} .
$$

Lemma A.1. Let $\eta: \mathbb{R} \rightarrow \mathbb{R}$ and $\beta, \gamma, T_{12}:(0, T) \times S_{\epsilon} \rightarrow \mathbb{R}$ be smooth, $\nu \in \mathbb{R}$, and suppose that

$$
\gamma_{t}+\beta \gamma_{x}^{2}\left(\eta^{\prime}(\gamma)-\nu \gamma_{x x}-T_{12}\right)=0, \quad \text { on }(0, T) \times S_{\epsilon}
$$

Let

$$
u:(0, T) \times S_{\epsilon} \rightarrow \mathbb{R}^{2} \hookrightarrow \mathbb{R}^{3}, \quad \text { and } \quad T:(0, T) \times S_{\epsilon} \rightarrow \mathbb{R}_{\text {sym }}^{2 \times 2} \hookrightarrow \mathbb{R}_{\text {sym }}^{3 \times 3},
$$

be smooth with $T_{12}$ as above, and let

$$
U=\nabla u-\left[\begin{array}{lll}
0 & \gamma & 0 \\
0 & 0 & 0 \\
0 & 0 & 0
\end{array}\right] \quad \text { and } \quad v_{d}=\left(I-\frac{\omega}{|\omega|} \otimes \frac{\omega}{|\omega|}\right) X(S \operatorname{Curl}(U))
$$


where

$$
S=\left(T-\left[\begin{array}{ccc}
0 & 2 \eta^{\prime}(\gamma) & 0 \\
0 & 0 & 0 \\
0 & 0 & 0
\end{array}\right]+2 \nu \operatorname{Curl}(\operatorname{Curl}(U))\right)_{\text {sym }} \quad \text { and } \quad \omega=X(\operatorname{Curl}(U))
$$

Then the triple $\left(U, u, v_{d}\right)$ satisfies equation (18).

Under the ansatz of the lemma the matrices $\operatorname{Curl}(U)$ and $\operatorname{Curl}(\operatorname{Curl}(U))$ become

$$
\operatorname{Curl}(U)=\left[\begin{array}{ccc}
0 & 0 & -\gamma_{x} \\
0 & 0 & 0 \\
0 & 0 & 0
\end{array}\right], \quad \operatorname{Curl}(\operatorname{Curl}(U))=\left[\begin{array}{ccc}
0 & -\gamma_{x y} & \gamma_{x x} \\
0 & 0 & 0 \\
0 & 0 & 0
\end{array}\right]
$$

and vectors $X(\operatorname{Curl}(U))$ and $v_{d}$ are

$$
X(\operatorname{Curl}(U))=\left(\begin{array}{c}
0 \\
-\gamma_{x} \\
0
\end{array}\right), \quad v_{d}=\left(\begin{array}{c}
\beta \gamma_{x}\left(\eta^{\prime}(\gamma)-\nu \gamma_{x x}-T_{12}\right) \\
0 \\
0
\end{array}\right) .
$$

Acknowledgement. The authors appreciate the insight gained from multiple discussions with Professor Amit Acharya of the Department of Civil and Environmental Engineering at Carnegie Mellon University.

\section{REFERENCES}

[1] Amit Acharya, New inroads in an old subject: plasticity, from around the atomic to the macroscopic scale, J. Mech. Phys. Solids 58 (2010), no. 5, 766-778, DOI 10.1016/j.jmps.2010.02.001. MR2642309

[2] Amit Acharya and Luc Tartar, On an equation from the theory of field dislocation mechanics, Boll. Unione Mat. Ital. (9) 4 (2011), no. 3, 409-444. MR2906769

[3] Amit Acharya and Xiaohan Zhang, From dislocation motion to an additive velocity gradient decomposition, and some simple models of dislocation dynamics, Chin. Ann. Math. Ser. B 36 (2015), no. 5, 645-658, DOI 10.1007/s11401-015-0970-0. MR3377868

[4] Giovanni Alberti, Guy Bouchitté, and Gianni Dal Maso, The calibration method for the MumfordShah functional and free-discontinuity problems, Calc. Var. Partial Differential Equations 16 (2003), no. 3, 299-333, DOI 10.1007/s005260100152. MR2001706

[5] L. Ambrosio and A. Braides, Energies in SBV and variational models in fracture mechanics, Homogenization and applications to material sciences (Nice, 1995), GAKUTO Internat. Ser. Math. Sci. Appl., vol. 9, Gakkōtosho, Tokyo, 1995, pp. 1-22. MR.1473974

[6] Luigi Ambrosio, Minimizing movements (Italian, with English and Italian summaries), Rend. Accad. Naz. Sci. XL Mem. Mat. Appl. (5) 19 (1995), 191-246. MR.1387558

[7] Pedro Areias and Ted Belytschko, Analysis of three-dimensional crack initiation and propagation using the extended finite element method, International Journal for Numerical Methods in Engineering 63 (2005), no. 5, 760-788.

[8] B. Bourdin, G. A. Francfort, and J.-J. Marigo, Numerical experiments in revisited brittle fracture, J. Mech. Phys. Solids 48 (2000), no. 4, 797-826, DOI 10.1016/S0022-5096(99)00028-9. MR/1745759

[9] Blaise Bourdin, Numerical implementation of the variational formulation for quasi-static brittle fracture, Interfaces Free Bound. 9 (2007), no. 3, 411-430, DOI 10.4171/IFB/171. MR2341850

[10] Blaise Bourdin, Gilles A. Francfort, and Jean-Jacques Marigo, The variational approach to fracture, J. Elasticity 91 (2008), no. 1-3, 5-148, DOI 10.1007/s10659-007-9107-3. MR2390547

[11] Gianni Dal Maso, An introduction to $\Gamma$-convergence, Progress in Nonlinear Differential Equations and their Applications, 8, Birkhäuser Boston, Inc., Boston, MA, 1993. MR1201152 
[12] Gianni Dal Maso, Antonio DeSimone, and Maria Giovanna Mora, Quasistatic evolution problems for linearly elastic-perfectly plastic materials, Arch. Ration. Mech. Anal. 180 (2006), no. 2, 237-291, DOI 10.1007/s00205-005-0407-0. MR2210910

[13] Gianni Dal Maso, Gilles A. Francfort, and Rodica Toader, Quasistatic crack growth in nonlinear elasticity, Arch. Ration. Mech. Anal. 176 (2005), no. 2, 165-225, DOI 10.1007/s00205-004-0351-4. MR.2186036

[14] Gianni Dal Maso and Rodica Toader, A model for the quasi-static growth of brittle fractures: existence and approximation results, Arch. Ration. Mech. Anal. 162 (2002), no. 2, 101-135, DOI 10.1007/s002050100187. MR:1897378

[15] Ennio De Giorgi, New problems on minimizing movements, Boundary value problems for partial differential equations and applications, RMA Res. Notes Appl. Math., vol. 29, Masson, Paris, 1993, pp. 81-98. MR.1260440

[16] John Dolbow and Ted Belytschko, A finite element method for crack growth without remeshing, Int. J. Numer. Meth. Eng 46 (1999), no. 1, 131-150.

[17] G. A. Francfort and J.-J. Marigo, Revisiting brittle fracture as an energy minimization problem, J. Mech. Phys. Solids 46 (1998), no. 8, 1319-1342, DOI 10.1016/S0022-5096(98)00034-9. MR1633984

[18] Gilles Francfort and Alexander Mielke, Existence results for a class of rate-independent material models with nonconvex elastic energies, J. Reine Angew. Math. 595 (2006), 55-91, DOI 10.1515/CRELLE.2006.044. MR.2244798

[19] Gilles A. Francfort and Christopher J. Larsen, Existence and convergence for quasi-static evolution in brittle fracture, Comm. Pure Appl. Math. 56 (2003), no. 10, 1465-1500, DOI 10.1002/cpa.3039. MR.1988896

[20] Morton E. Gurtin, Eliot Fried, and Lallit Anand, The mechanics and thermodynamics of continua, Cambridge University Press, Cambridge, 2010. MR2884384

[21] J.P. Hirth and J. Lothe, Theory of dislocations, Krieger Publishing Company, 1982.

[22] H Horii and Siavouche Nemat-Nasser, Brittle failure in compression: splitting, faulting and brittleductile transition, Philosophical Transactions of the Royal Society of London A: Mathematical, Physical and Engineering Sciences 319 (1986), no. 1549, 337-374.

[23] A. Mesgarnejad, B. Bourdin, and M. M. Khonsari, Validation simulations for the variational approach to fracture, Comput. Methods Appl. Mech. Engrg. 290 (2015), 420-437, DOI 10.1016/j.cma.2014.10.052. MR3340163

[24] A. Mielke, Energetic formulation of multiplicative elasto-plasticity using dissipation distances, Contin. Mech. Thermodyn. 15 (2003), no. 4, 351-382, DOI 10.1007/s00161-003-0120-x. MR1999280

[25] Alexander Mielke and Florian Theil, On rate-independent hysteresis models, NoDEA Nonlinear Differential Equations Appl. 11 (2004), no. 2, 151-189, DOI 10.1007/s00030-003-1052-7. MR2210284

[26] R. E. Showalter, Hilbert space methods for partial differential equations, Pitman, London-San Francisco, Calif.-Melbourne, 1977. Monographs and Studies in Mathematics, Vol. 1. MR0477394

[27] R. E. Showalter, Monotone operators in Banach space and nonlinear partial differential equations, Mathematical Surveys and Monographs, vol. 49, American Mathematical Society, Providence, RI, 1997. MR 1422252

[28] Augusto Visintin, On the homogenization of visco-elastic processes, IMA J. Appl. Math. 77 (2012), no. 6, 869-886, DOI 10.1093/imamat/hxs055. MR.2999142

[29] Xiaohan Zhang, Amit Acharya, Noel J. Walkington, and Jacobo Bielak, A single theory for some quasi-static, supersonic, atomic, and tectonic scale applications of dislocations, J. Mech. Phys. Solids 84 (2015), 145-195, DOI 10.1016/j.jmps.2015.07.004. MR3413434 